\title{
Searching for GeV-scale Majorana Dark Matter: inter spem et metum
}

\author{
Adil Jueid, ${ }^{a}$ Salah Nasri ${ }^{b, c}$ and Rachik Soualah ${ }^{d, c}$ \\ ${ }^{a}$ Department of Physics, Konkuk University, \\ Seoul 05029, Republic of Korea \\ ${ }^{b}$ Department of physics, United Arab Emirates University, \\ Al-Ain, UAE \\ ${ }^{c}$ The Abdus Salam International Centre for Theoretical Physics, \\ Strada Costiera 11, I-34014, Trieste, Italy \\ ${ }^{d}$ Department of Applied Physics and Astronomy,University of Sharjah, \\ P.O. Box 27272, Sharjah, UAE \\ E-mail: adil.hep@gmail.com, salah.nasri@gmail.com, \\ rsoualah@sharjah.ac.ae
}

Abstract: We suggest a minimal model for GeV-scale Majorana Dark Matter (DM) coupled to the standard model lepton sector via a charged scalar singlet. We show that there is an anti-correlation between the spin-independent DM-Nucleus scattering cross section $\left(\sigma_{\mathrm{SI}}\right)$ and the DM relic density for parameters values allowed by various theoretical and experimental constraints. Moreover, we find that even when DM couplings are of order unity, $\sigma_{\mathrm{SI}}$ is below the current experimental bound but above the neutrino floor. Furthermore, we show that the considered model can be probed at high energy lepton colliders using e.g. the mono-Higgs production and same-sign charged Higgs pair production.

Keywords: Beyond Standard Model, Higgs Physics

ArXiv EPrint: 2006.01348 


\section{Contents}

1 Introduction 1

2 The model 2

3 Theoretical and experimental constraints 4

4 LHC constraints $\quad 6$

5 Dark Matter phenomenology 1

6 Prospects at the International Linear Collider 11

$\begin{array}{lll}7 & \text { Conclusions } & 16\end{array}$

$\begin{array}{ll}\text { A Computation of the effective } \tilde{y}_{H N N} \text { coupling } & 18\end{array}$

B LHC limits: Signal Regions (SR) and the statistical treatment 19

\section{Introduction}

The existence of Dark Matter (DM) in the universe is an established fact supported by various observations at the sub-galactic, galactic, and cosmological scales (for a review, see e.g. [1]). The measurements of CMB anisotropies implied that DM constitutes about $80 \%$ of the matter budget in the universe with density of $\Omega_{\mathrm{DM}} h^{2}=0.1198 \pm 0.0015$ [2], and the standard theories of structure formation requires it to be non-relativistic when gravitational clustering started at the matter-radiation equality. This type of DM is known as cold DM (CDM). One of the simplest scenarios of CDM is the thermal-freeze out mechanism in which the DM can be accommodated by Weakly Interacting Massive Particles (WIMPs) produced in thermal bath and as the universe expands and cools down their relic abundance freeze-out when temperature drops below their mass. Possible evidence for WIMP DM has driven numerous experimental efforts to search for it via direct detection $[3,4]$, indirect detection [5-8] and in collider experiments [9, 10]. Unfortunately, no signal for CDM has been detected, and consequently upper limit on the cross section of their scattering off nucleus in the mass range $\mathrm{GeV}$ to $\mathrm{TeV}$ are obtained, with the stringent bound of $4.1 \times 10^{-47} \mathrm{~cm}^{2}$ for a mass of $30 \mathrm{GeV}$. It is expected that the upcoming XENONnT experiment will be able to probe cross sections smaller by more than an order of magnitude [11]. Moreover, a model-independent estimate of the limits on the annihilation cross sections of WIMP DM implies strong constraints on models with $s$-wave annihilation channel [12]. These strong exclusions combined with the null results from direct detection experiments 
put an end to the most minimal model for GeV-scale DM candidate, i.e. the SM with real scalar singlet $[13,14]$ which called for several extensions $[15,16]$.

Models with a singlet Majorana fermion as a DM candidate can potentially avoid these constraints due to the fact that their annihilation is dominated by $p$-wave amplitudes (which are suppressed by the square of the DM velocity) in addition of being both minimal and predictive. On the other hand, the scattering of the Majorana DM off the nucleus is induced at the one-loop order due to the absence of tree level couplings of the Majorana $\mathrm{DM}$ to the $Z / H$ bosons. Consequently, models containing Majorana DM can evade easily direct detection constraints even for model parameters of order $\mathcal{O}(1)$ and hence avoiding the over-abundance condition $\Omega_{\mathrm{WIMP}} h^{2} \leqslant \Omega_{\text {Planck }} h^{2}$. Models containing Majorana DM are phenomenologically very attractive as they can easily address the question of the smallness of neutrino mass through radiative mass generation mechanism, and the problem of baryon asymmetry in the universe (BAU) via electroweak baryogenesis [17-22]. In this work, we suggest a very simple model which extends the Standard Model (SM) particle content by two gauge singlets: a charged scalar and a Majorana fermion. Using simple correlations between the relic abundance and the predicted spin-independent cross section, we show that the model is neither excluded nor close to the neutrino-floor region. We study the potential discovery of the DM and the charged Higgs in both $e^{-} e^{-}$and $e^{-} e^{+}$processes. We find that it can be exclusively probed at lepton colliders such as the international linear collider (ILC) while the current and future LHC constraints will play a complementary role.

The remainder of this paper is organized as follows: in section 2, we discuss briefly the model's new degrees of freedom and their possible interactions. In section 3, we study the various theoretical and experimental constraints on parameter space of the model; in particular, we study constraints from perturbative unitarity, boundness-from-below of the scalar potential, $\mathrm{BR}(H \rightarrow \gamma \gamma)$ measurements, and the impact from bounds from leptonflavor violating decays and Higgs boson invisible decays. In section 4, we discuss of the impact of searches of charginos/sleptons at the LHC on the parameter space of our model. In section 5, we discuss in detail the DM phenomenology of our model with emphasis on the relic abundance of DM candidate, and its correlation to the spin-independent DMNucleus cross section. The collider implications are discussed in section 6. We summarize our results in section 7 . Some technical details are reported in appendices A and B.

\section{The model}

We consider a minimal extension of the SM which contains, in addition to the SM particle content, two gauge-singlet fields; a charged scalar $S$ and a right handed (RH) neutral fermion $N$. Under the $\mathrm{SM}$ gauge group $\mathrm{SU}(3) \otimes \mathrm{SU}(2)_{L} \otimes \mathrm{U}(1)_{Y}$, the two new degrees of freedom transform as

$$
S:(\mathbf{1}, \mathbf{1}, \mathbf{2}), \quad \text { and } \quad N:(\mathbf{1}, \mathbf{1}, \mathbf{0}) \text {. }
$$

Moreover, to ensure the stability of DM in the universe, we impose an exact discrete symmetry $Z_{2}$ under which all the SM fields are even while the new states are odd, i.e. 
$\left\{V^{\mu}, \Phi, \ell, q\right\} \rightarrow\left\{V^{\mu}, \Phi, \ell, q\right\}$, and $\{S, N\} \rightarrow\{-S,-N\}$, with the condition that $N$ is always lighter than the charged scalar singlet. In this simple model, the $\mathrm{RH}$ fermion plays the role of the DM candidate while the charged scalar is a mediator of the DM-visible interaction. Under these symmetry requirements, the most general Yukawa Lagrangian can be written as ${ }^{1}$

$$
\mathcal{L}_{\text {Yuk }} \supset \sum_{\ell=e, \mu, \tau} y_{\ell} \bar{\ell}_{R}^{c} S N_{R}+\frac{1}{2} M_{N} \bar{N}_{R}\left(N_{R}\right)^{c}+\text { h.c. }
$$

The most general CP-invariant, and dimension four potential involving the SM Higgs doublet $\Phi$ and the charged Higgs scalar $S$ can be written as

$$
V(\Phi, S)=-m_{11}^{2}\left|\Phi^{\dagger} \Phi\right|+m_{22}^{2}\left|S^{\dagger} S\right|+\lambda_{1}\left|\Phi^{\dagger} \Phi\right|^{2}+\lambda_{2}\left|S^{\dagger} S\right|^{2}+\lambda_{3}\left|\Phi^{\dagger} \Phi\right|\left|S^{\dagger} S\right|,
$$

with $m_{11}^{2}, m_{22}^{2}, \lambda_{i, i=1,2,3}$ are real parameters, and $\Phi$ is the SM Higgs doublet parametrised as

$$
\Phi=\left(\begin{array}{c}
\omega^{+} \\
\frac{1}{\sqrt{2}}\left(v+H+i \omega^{0}\right)
\end{array}\right) .
$$

After the electroweak symmetry breaking, the pseudo-Goldstone bosons $\left(\omega^{0, \pm}\right)$ get absorbed by the $Z / W^{ \pm}$gauge bosons, and one lefts with three new scalars; a CP-even scalar $H$ which is identified with the recently discovered $125 \mathrm{GeV}$ SM Higgs boson and a pair of charged scalars $H^{ \pm}$. Their tree-level masses are given by:

$$
m_{H}^{2}=\lambda_{1} v^{2}, \quad \text { and } \quad m_{H^{ \pm}}^{2}=m_{22}^{2}+\frac{1}{2} \lambda_{3} v^{2} .
$$

The non-trivial transformation of the scalar singlet under $\mathrm{U}(1)_{Y}$ allows for gauge interactions with the photon and $Z$-boson. The gauge interaction of the $S$ state is given by

$$
\begin{aligned}
\mathcal{L}_{\text {gauge }}= & -i\left(e A^{\mu}-e \tan \theta_{W} Z^{\mu}\right)\left[S^{\dagger}\left(\partial_{\mu} S\right)-\left(\partial_{\mu} S^{\dagger}\right) S\right] \\
& +e^{2} A_{\mu} A^{\mu} S^{\dagger} S+e^{2} \tan ^{2} \theta_{W} Z_{\mu} Z^{\mu} S^{\dagger} S-2 e^{2} \tan \theta_{W} A_{\mu} Z^{\mu} S^{\dagger} S .
\end{aligned}
$$

In addition to the SM parameters, the model includes the following seven independent parameters

$$
\left\{m_{H^{ \pm}}, m_{N}, \lambda_{2}, \lambda_{3}, y_{e}, y_{\mu}, y_{\tau}\right\}
$$

For convenience, we define $y_{N}$ as the combination of the new Yukawa couplings as:

$$
y_{N}=\sqrt{y_{e}^{2}+y_{\mu}^{2}+y_{\tau}^{2}}
$$

\footnotetext{
${ }^{1} h . c=\sum_{\ell=e, \mu, \tau} y_{\ell}^{*} \bar{N}_{R}^{c} S^{*} \ell_{R}+\frac{1}{2}(\bar{N})_{R}^{c} M_{N}^{*} N_{R}$. In the case of more than one right handed neutrino, the second term has the same form with $M_{N}$ a symmetric matrix. Note that

$$
N_{R}=\frac{1}{2}\left(1+\gamma_{5}\right) \Psi_{D},\left(N_{R}\right)^{c}=\frac{1}{2}\left(1-\gamma_{5}\right) \Psi_{D}
$$

where $\Psi_{D}$ is a 4 components spinor (i.e. Dirac spinor; which one could denote by just $N$ ).
} 


\section{Theoretical and experimental constraints}

The parameters of the model are subject to various theoretical and experimental constraints. Due to the fact that the charged Higgs state is gauge singlet, constraints from direct LHC searches are mainly coming from dilepton plus missing energy searches that we will discuss in the next section. Besides, the SM Higgs boson is really SM-like, i.e. there is no modification of tree-level Higgs couplings to fermions and gauge bosons. Therefore, the only modification to the Higgs boson decay rates comes from the effect of the charged Higgs boson on the one-loop induced $H \rightarrow \gamma \gamma$ decay width [23-25]. In our model, the partial decay width of $H \rightarrow \gamma \gamma$ is given by [23]

$$
\Gamma(H \rightarrow \gamma \gamma)=\frac{G_{F} \alpha^{2} m_{H}^{3}}{128 \sqrt{2} \pi^{3}}\left|\sum_{f=\tau, b, t} N_{c, f} Q_{i}^{2} A_{1 / 2}\left(\tau_{f}^{2}\right)+A_{1}\left(\tau_{W}^{2}\right)+\frac{\lambda_{3} v^{2}}{2 m_{H^{ \pm}}^{2}} A_{0}\left(\tau_{H^{ \pm}}^{2}\right)\right|^{2},
$$

with $\tau_{i}=2 m_{i} / m_{H}, N_{c f}$ is the number of colors for the fermion $f$, and $A_{i}\left(\tau_{i}\right)$ are the oneloop form factors which can be found in [23]. In the SM, the contribution of the $W$-boson is dominant and has a reversed sign as compared to the fermionic one. Adding the charged Higgs boson modify drastically the rate of $H \rightarrow \gamma \gamma$ wherein the new contribution is mainly controlled by the value of $\lambda_{3}$. We note that constructive (destructive) interference effects, within the SM contributions, occur for negative (positive) values of $\lambda_{3}$. Therefore, we expect that the enhancement of $\kappa_{\gamma}$ always occur for $\lambda_{3}<0$ while for $\lambda_{3}>0$ we may have three different regimes: (i) large charged Higgs boson contribution that overcomes that of the $W$-boson and implies an enhancement of $\kappa_{\gamma}$, (ii) positive but small contribution of the charged Higgs boson which makes $\kappa_{\gamma}$ consistent with the experimental measurement and (iii) exact or almost exact cancellation between the charged Higgs boson and the $W$-boson contributions which make $\kappa_{\gamma}$ very small. In this work, we use the ATLAS-CMS combined measurement of $\left|\kappa_{\gamma}\right|=\sqrt{\Gamma(H \rightarrow \gamma \gamma) / \Gamma(H \rightarrow \gamma \gamma)_{\mathrm{SM}}}=0.87_{-0.09}^{+0.14}[26]$.

In addition to constraint from the signal strength measurement, the model should conform to a number of theoretical constraints such as ${ }^{2}$

- The quartic couplings of the scalar potential should satisfy the vacuum stability conditions (or boundedness-from-below) [33]. These conditions can be written as: $\lambda_{1,2}>0, \lambda_{3}+2 \sqrt{\lambda_{1} \lambda_{2}}>0$.

- The perturbative unitarity should be maintained in various scattering-processes at high-energy $[34,35]$. This requirement can be translated to a set of conditions on the combinations of the quartic couplings, i.e. $\max \left\{e_{1}, e_{2}, e_{3}\right\}<4 \pi$, where $e_{i}$ represents the different eigenvalues of the scattering matrices given by $e_{1}=\lambda_{3}$ and $e_{2,3}=$ $-3\left(\lambda_{1}+\lambda_{2}\right) \pm \sqrt{9\left(\lambda_{1}+\lambda_{2}\right)^{2}+4 \lambda_{3}}$.

- The electroweak vacuum should be a global minimum [36] which can be satisfied for

$$
\frac{m_{11}^{2}}{\sqrt{\lambda_{1}}} \geq-\frac{m_{22}^{2}}{\sqrt{\lambda_{2}}}
$$

\footnotetext{
${ }^{2}$ Note that the theoretical constraints on our model can be obtained from those in the Inert Higgs Doublet Model (IHDM) by simply setting $\lambda_{4}=\lambda_{5}=0$ (see e.g. [32]).
} 

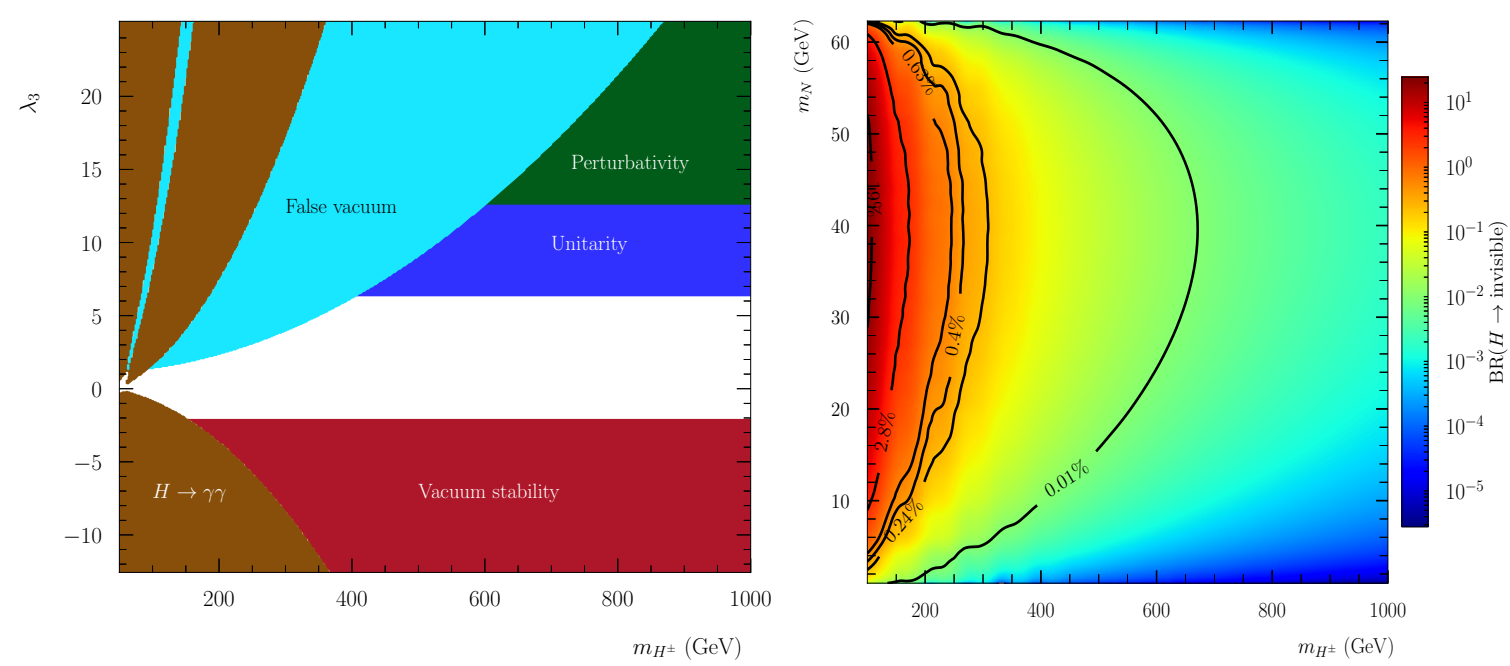

Figure 1. Left: summary of the impact of theoretical and experimental constraints on the parameter space of the scalar sector of the model. The constraints are depicted in the mass of the charged Higgs boson $m_{H^{ \pm}}$and $\lambda_{3}$ for $\lambda_{2}=2$. The excluded regions are shown for constraints from $H \rightarrow \gamma \gamma$ (brown), from false vacuum (cyan), from vacuum stability (red), from perturbativity (green), and from perturbative unitarity (blue). The white shaded area corresponds to the allowed region of the 2D parameter space. Right: the Higgs boson invisible decay branching projected in the plane of the mass of the charged Higgs $m_{H^{ \pm}}$and the mass of the Majorana DM $m_{N}$. The contours in solid black lines show the expected limits from HL-LHC [27], FCC-ee [28], ILC [29], CEPC [30] and FCC-hh [31] and correspond to upper bounds on $\mathrm{BR}(H \rightarrow$ invisible) ranging from $2.8 \%$ to $0.01 \%$.

A summary of the theoretical and experimental constraints are shown in the left panel of figure 1. The combination of all these constraints restricts the allowed range of $\lambda_{3}$ and the charged Higgs boson mass; namely $\lambda_{3}$ cannot be larger than 5 for all the charged Higgs masses. The constraints on the Yukawa coupling $y_{N}$ come mainly from the limit on branching fraction of the Higgs boson invisible decay mode; $\mathrm{BR}(H \rightarrow$ invisble $) \equiv$ $B_{\text {inv }}$. This decay occurs at the one-loop order, which we have computed using FeynArts, FormCalc and LoopTools [37, 38]. The strongest and up-to-date stringent bound on $\mathrm{B}_{\text {inv }}$ was reported by the CMS collaboration using a combination of previous Higgs to invisible decay searches at 7,8 and $13 \mathrm{TeV}$, where it has been found $B_{\text {inv }}<B_{\text {upper }}=0.19$ at $95 \%$ CL [39] assuming SM production rates of the Higgs boson. Using the results of these searches, we derive an upper bound on the magnitude of $y_{N}$

$$
y_{N}<\left(\frac{2048 \pi^{5} \Gamma_{H}^{\mathrm{SM}}}{\beta_{N}^{3 / 2} m_{H} \lambda_{3}^{2} v^{2} m_{N}^{2}\left|C_{0}+C_{2}\right|^{2}\left(\frac{1}{B_{\text {upper }}}-1\right)}\right)^{1 / 4},
$$

with $\beta_{N}=\left(1-4 m_{N}^{2} / m_{H}^{2}\right)$, and $C_{0,2} \equiv C_{0,2}\left(m_{N}^{2}, m_{H}^{2}, m_{N}^{2}, m_{\ell}^{2}, m_{H^{ \pm}}^{2}, m_{H^{ \pm}}^{2}\right)$ are the PassarinoVeltman functions [40]. For instance, for $m_{N} \simeq 40 \mathrm{GeV}, \lambda_{3}=1$ and $m_{H^{ \pm}}=200 \mathrm{GeV}$, we get $y_{N}<2.5$. In the right panel of figure 1 , we illustrate the predicted values of the $\mathrm{BR}(H \rightarrow$ invisible $)$ projected on the $\left(m_{H^{ \pm}}, m_{N}\right)$ plane for $\lambda_{3}=1$ and $y_{N}=2$. In the same panel, we show the corresponding contours of the expected bounds from HLLHC [27], FCC-ee [28], ILC [29], CEPC [30] and FCC-hh [31]. We can see that the 
current LHC bounds do not play a significant role in restricting the $2 \mathrm{D}$ parameter space. However, the expected bounds from FCC-hh can exclude charged masses up to $600 \mathrm{GeV}$ for $m_{N} \in[40,50] \mathrm{GeV}$.

The experimental collaborations at LEP have carried out several searches of new physics scenarios such as the chargino and neutralino production at center-of-mass energies $\sqrt{s}=183-209 \mathrm{GeV}$. For instance, the OPAL collaboration has searched for new physics signal in $\ell^{+} \ell^{-}+E_{T}^{\text {miss }}$ final state using $680 \mathrm{pb}^{-1}$ of luminosity [41] whose null results can be used to put stringent bounds on the masses and couplings of charged scalars. In contrast, these obtained constraints do not affect significantly the model-parameter space; we found $m_{H^{ \pm}}>90 \mathrm{GeV}$ for $y_{N} \simeq 1-2$; for more details, see e.g. [42, 43].

It is worth mentioning that constraints from lepton-flavor violating decays on the $y_{\ell}$ couplings are very important as it will be discussed below. The most stringent bound comes from the branching ratio of $\mu \rightarrow e \gamma$ decay. Using the most recent experimental bounds on $\operatorname{BR}\left(\ell_{\alpha} \rightarrow \ell_{\beta} \gamma\right)$ from the MEG [44] and BABAR [45] experiments, we derive the following bounds:

$$
\begin{aligned}
\left|y_{e} y_{\mu}\right|<\left(\frac{2.85 \times 10^{-5}}{\mathrm{GeV}}\right)^{2} \frac{m_{H^{ \pm}}^{2}}{\left|\mathcal{F}\left(m_{N}^{2} / m_{H^{ \pm}}^{2}\right)\right|}, \\
\left|y_{e} y_{\tau}\right|<\left(\frac{2.87 \times 10^{-4}}{\mathrm{GeV}}\right)^{2} \frac{m_{H^{ \pm}}^{2}}{\left|\mathcal{F}\left(m_{N}^{2} / m_{H^{ \pm}}^{2}\right)\right|}, \\
\left|y_{\tau} y_{\mu}\right|<\left(\frac{3.07 \times 10^{-4}}{\mathrm{GeV}}\right)^{2} \frac{m_{H^{ \pm}}^{2}}{\left|\mathcal{F}\left(m_{N}^{2} / m_{H^{ \pm}}^{2}\right)\right|} .
\end{aligned}
$$

Here $\mathcal{F}(X)$ is the one-loop function which can be found in [46, 47]. The implications of these constraints on the product of the couplings are very important. For example, if we fix $y_{e}=2$, we found that $\left\{y_{e} y_{\mu}, y_{\tau} y_{e}, y_{\tau} y_{\mu}\right\}<\left\{1.5-6.7 \times 10^{-4}, 0.05-0.1,0.20-1.82 \times 10^{-5}\right\} .^{3}$ In the following section, we consider an electron-specific scenario where we choose $y_{e}=2$, $y_{\mu}=10^{-4}$ and $y_{\tau}=10^{-1}$.

\section{LHC constraints}

The Yukawa interaction in eq. (2.2) is subject to constraints from LHC searches of sleptons. This is due to the fact that for such interaction, the charged Higgs boson can be pair produced through $q \bar{q}$ annihilation with the exchange of $\gamma^{*} / Z$-bosons and its decay products lead to $2 \ell+E_{T}^{\text {miss }}$ final state. In order to explore the constraining power of the LHC, we take into account the most recent search for electroweak production of supersymmetric particles (sleptons/charginos) by the ATLAS collaboration at $\sqrt{s}=13 \mathrm{TeV}$ with a recorded luminosity $\mathcal{L}=139 \mathrm{fb}^{-1}$ [48]. For this purpose, exclusive samples of charged Higgs boson pair production have been generated at the Lowest Order (LO) with up to extra partons in the final state using Madgraph_aMC@NLO [49]. The MC signal samples were generated

\footnotetext{
${ }^{3}$ Different scenarios where we can have $y_{e} \simeq y_{\mu} \simeq y_{\tau} \simeq 10^{-3}$ or $y_{\tau} \simeq y_{\mu} \gg y_{e} \simeq 10^{-4}$ are possible as well, but none of these scenarios are phenomenologically plausible since the production rates at lepton colliders will be extremely small - the cross sections depend exclusively on $y_{e}$. Theoretically, it could be possible to have a discrete flavor symmetry which allows for $y_{e} \simeq \mathcal{O}(1) \gg y_{\tau} \gg y_{\mu}$.
} 
using a UFO format model file [50]. The hard-scattering parton-level matrix elements are convoluted with the NNPDF3.0_as_0118_10 [51] with $\alpha_{s}\left(M_{Z}^{2}\right)=0.118$ using the LHAPDF library [52]. The MC signal events were decayed using MadSpin [53]. The merging of the exclusive samples were performed using the MLM merging scheme [54] in addition to Pythia8 [55] using a merging scale that it is equal to the quarter of the charged higgs boson mass. Moreover, jets are clustered using FastJet [56] with the anti- $k_{T}$ algorithm with the jet radius parameter of $R=0.4$ [57]. The merged cross section values vary from $\sigma_{p p \rightarrow H^{+} H^{-} j j}=81.12 \mathrm{fb}$ for $m_{H^{ \pm}}=100 \mathrm{GeV}$ to $\sigma_{p p \rightarrow H^{+} H^{-} j j}=0.17 \mathrm{fb}$ for $m_{H^{ \pm}}=500 \mathrm{GeV}$. Our results were found to be in excellent agreement with the slepton pair production at the ATLAS collaboration that reported $\sigma_{p p \rightarrow \tilde{\ell} \tilde{\ell}}=0.18 \pm 0.01 \mathrm{fb}$ for $m_{\tilde{\ell}}=500 \mathrm{GeV}$.

The ATLAS search strategy is based on selecting events that have exactly two oppositely charged signal leptons, with $p_{T}>25 \mathrm{GeV}$. All jets are predefined with $p_{T}>20 \mathrm{GeV}$ and $|\eta|<2.4$. Furthermore, events are then considered if the lepton pairs invariant mass $m_{\ell \ell}>100 \mathrm{GeV}$. The passed events have to fulfill another additional requirement where no reconstructed $b$-jets are selected in each event. These events satisfy extra requirements where $E_{T}^{\text {miss }}>110 \mathrm{GeV}$ and MET significance $>10$. Additional constraint is explored by studying the stransverse mass $M_{T 2}[58,59]$, defined as

$$
M_{T 2}=\min _{q_{T}^{(1)}+q_{T}^{(2)}=p_{T}^{\text {miss }}}\left\{\max \left\{M_{T}\left(p_{T}^{(1)}, q_{T}^{(1)}\right), M_{T}\left(p_{T}^{(2)}, q_{T}^{(2)}\right)\right\}\right\}
$$

where $p_{T}^{(1)}$ and $p_{T}^{(2)}$ are the three-momentum of the leading and subleading charged leptons, respectively; whereas $q_{T}^{(1)}$ and $q_{T}^{(2)}$ represent the three-momentum vectors of the unknown particles which can be combined in one vector $\mathbf{q}_{T}^{(1)}+\mathbf{q}_{T}^{(2)}=\mathbf{p}_{T}^{\text {miss }}$. The transverse mass in this case is given by $M_{T}\left(p_{T}^{i}, q_{T}^{i}\right)=\sqrt{2 \times\left|p_{T}^{i}\right| \times\left|q_{T}^{i}\right| \times(1-\cos \Delta \phi)}$. The $M_{T 2}$ kinematical variable can be used to fasten the masses of the particles pair that can possibly escape the detection in each selected event. Hence, this will lead to reliable discrimination of the signal regions (SR). Depending on the topology of the events, they will be divided into two categories: same flavor (SF) events (where $m_{\ell \ell}>121.2 \mathrm{GeV}$ cut is imposed) and different Flavor (DF) events. Moreover, an extra event classification is taken into account based on the multiplicity of non- $b$-tagged jets $\left(n_{\mathrm{j}}\right)$ in each events where its content could have $n_{\mathrm{j}}=0$ or $n_{\mathrm{j}}=1$. In our model, the charged Higgs decays branching ratios are: $\mathrm{BR}_{H^{ \pm} \rightarrow e^{ \pm} N}=99.76 \%, \mathrm{BR}_{H^{ \pm} \rightarrow \tau^{ \pm} N}=2.33 \times 10^{-3}$, and $\mathrm{BR}_{H^{ \pm} \rightarrow \mu^{ \pm} N} \simeq 3.58 \times 10^{-9}$. Therefore, the strongest limits come from the signal region with SF leptons and $n_{\mathrm{j}}=0$ or $n_{\mathrm{j}}=1$, where these regions are electron enriched. For this purpose, the MadAnalysis 5 framework [60-64] is used to obtain the constraints from the reinterpretation of this ATLAS search. The $\mathrm{CL}_{s}$ value for each point in the parameter space is computed to obtain the exclusion bound at the 95\% confidence level [65] (more details can be found in appendix B), and in figure 2 we give projected values on the $\left(m_{H^{ \pm}}, m_{N}\right)$ plane. The strongest limit comes from the signal region with $\mathrm{SF}, n_{\mathrm{j}}=0$ and $M_{T 2} \in[160, \infty)$. Furthermore, charged Higgs masses up to $\simeq 400 \mathrm{GeV}$ can be excluded at 95\% CL considering the actual search (the additional limits from different signal regions are described in appendix B). 


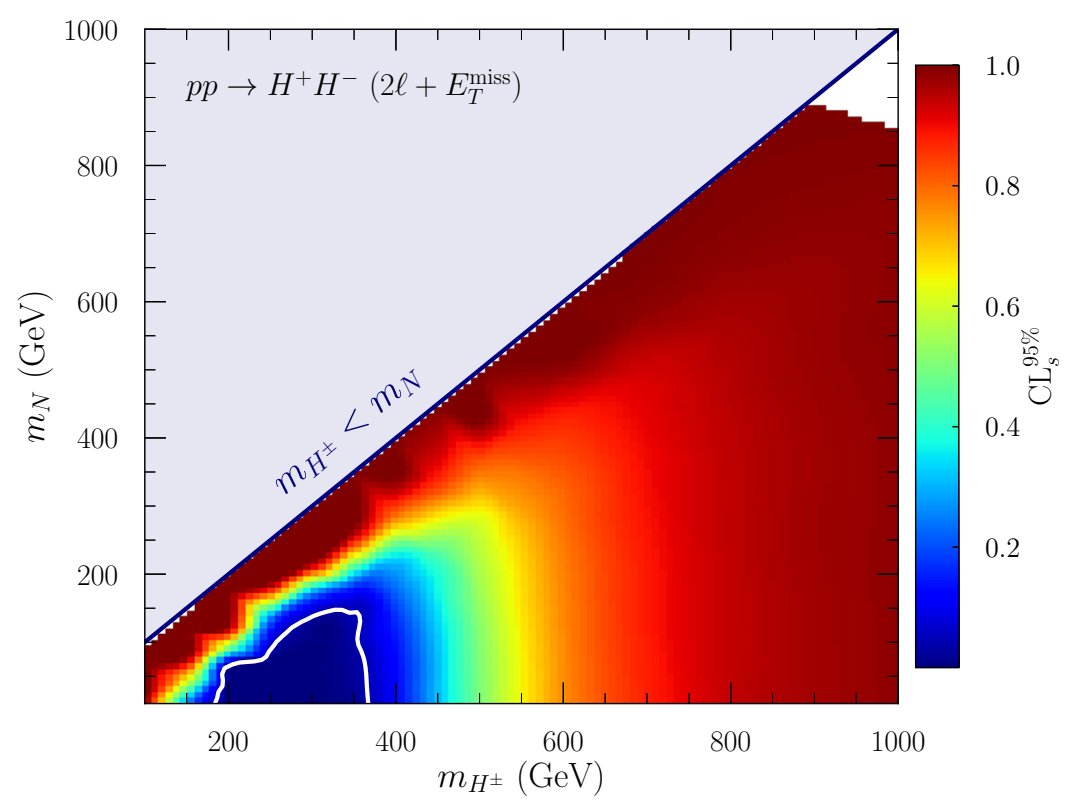

Figure 2. The LHC exclusions from slepton searches at $\sqrt{s}=13 \mathrm{TeV}$ and $\mathcal{L}=139 \mathrm{fb}^{-1}$. The limits are projected on the mass of the charged Higgs and the mass of the Majorana DM. The white solid line corresponds to $\mathrm{CL}_{s}=0.05$ below which the parameter point is excluded at the $95 \%$ CL.

\section{Dark Matter phenomenology}

The relic density of the $N$ particle can be approximated as follows [66]:

$$
\Omega_{\mathrm{DM}} h^{2} \simeq \frac{3 \times 10^{-26} \mathrm{~cm}^{3} \mathrm{~s}^{-1}}{\left\langle\sigma_{\mathrm{eff}}\left(x_{f}\right)\right\rangle}
$$

where $\sigma_{\text {eff }}\left(x_{f}\right)$ is the effective annihilation cross section at $x_{f}=m_{N} / T_{f}$ with $T_{f}$ being the freeze out temperature. The effective cross section is given by

$$
\sigma_{\mathrm{eff}}\left(x_{f}\right)=\sum_{i, j}^{2} \frac{4}{g_{\mathrm{eff}}^{2}}\left(1+\Delta_{i}\right)^{3 / 2}\left(1+\Delta_{j}\right)^{3 / 2} e^{-x_{f}\left(\Delta_{i}+\Delta_{j}\right)}\left\langle\sigma_{i j} v_{r}\right\rangle .
$$

In eq. (5.2), $g_{\text {eff }}$ refers to the effective number of degrees of freedom $g_{\text {eff }}\left(x_{f}\right)=\sum_{i=1}^{2} 2(1+$ $\left.\Delta_{i}\right)^{3 / 2} e^{-x_{f} \Delta_{i}}, \sigma_{i j}$ refers to all the possible annihilation and co-annihilation cross sections and $\Delta_{i}$ is the relative mass splitting defined by $\Delta_{i}=\left(M_{i}-m_{N}\right) / m_{N}\left(M_{1}=m_{N}, M_{2}=\right.$ $\left.m_{H^{ \pm}}\right)$. In our analysis, we have included all possible annihilation and co-annihilation processes. For the annihilation, there are two major contributions: (i) $N N \rightarrow \ell^{+} \ell^{-}$from the exchange of the charged Higgs boson in $t$ - and $u$-channels, and (ii) $N N \rightarrow \sum X \bar{X}$ (the sum is over all the SM particles) which arises from the exchange of the SM Higgs boson via the $s$-channel. In addition, the co-annihilation channels involve two generic contributions: (i) $N H^{ \pm} \rightarrow \ell^{ \pm} H, W^{ \pm} \nu, \ell^{ \pm} Z, \ell^{ \pm} \gamma$ and (ii) $H^{ \pm} H^{\mp} \rightarrow \ell^{ \pm} \ell^{\mp}, q \bar{q}, H H, Z Z, W^{ \pm} W^{\mp}, Z H, t \bar{t}$. The main contribution of the $N$ particles to the relic density comes from their annihilation into charged leptons via the exchange of a charged scalar in the $t$ - and $u$-channel. Moreover, the contribution of $s$-channel diagrams to the relic density is one-loop induced, and therefore 
is sub-leading which makes it below $1 \%$ of the total contribution. Note that the annihilation rate in the s-channel does not depend significantly on $\lambda_{3}$ parameter if the later had to satisfy the perturbativity bound. For small mass-splittings, i.e. $\Delta=\left(m_{H^{ \pm}}-m_{N}\right) / m_{N}<0.1$, we find that co-annihilations become very important and for some values of the model parameters (notably for $y_{N}<1$ ) dominates over the annihilation processes.

Now, we consider the scattering of the $N$ particles off a nuclei $(\mathcal{N})$, and since the which occurs at the one-loop level through the exchange of the SM Higgs boson. The corresponding spin-independent cross section is given by

$$
\sigma_{\mathrm{SI}}=\frac{4}{\pi} \mu_{\mathcal{N}}^{2}\left(Z \cdot f_{p}+(A-Z) \cdot f_{n}\right)^{2}
$$

Here $f_{p, n}$ are the nucleons $(p / n)$ spin-independent form factors, and $\mu_{\mathcal{N}} \equiv m_{N} m_{\mathcal{N}} /\left(m_{N}+\right.$ $\left.m_{\mathcal{N}}\right)$ is the reduced mass of the DM- $\mathcal{N}$ system. The effective form-factors $\left(f_{p, n}\right)$ are connected to the matrix element of the $q N$ scattering by:

$$
f_{p}=m_{p} \sum_{u, d, s} \frac{\mathcal{A}_{q}}{m_{q}} f_{p}^{S q}+\frac{2}{27} m_{p} f_{p}^{S g} \sum_{c, b, t} \frac{\mathcal{A}_{q}}{m_{q}} .
$$

For the proton, $\mathcal{A}_{q}$ is related to the leading-order matrix elements by

$$
\mathcal{M}_{q N \rightarrow q N}=\mathcal{A}_{q}\left\langle p\left|\bar{\psi}_{q} \psi_{q}\right| p\right\rangle
$$

where

$$
\left\langle p\left|\bar{\psi}_{q} \psi_{q}\right| p\right\rangle= \begin{cases}\frac{m_{p}}{m_{q}} \cdot f_{p}^{S q}, & \text { for } q=u, d, s \\ \frac{2}{27} \frac{m_{p}}{m_{q}} \cdot f_{p}^{S g}, & \text { for } q=c, b, t\end{cases}
$$

and

$$
\mathcal{A}_{q}=\frac{\tilde{y}_{H N N}}{m_{H}^{2}} \cdot \frac{m_{q}}{v} \bar{\psi}_{N} \psi_{N},
$$

the effective $\tilde{y}_{H N N}$ is calculated at the one-loop order (see section A for more details)

$$
\tilde{y}_{H N N} \simeq-\frac{\lambda_{3} v\left|y_{N}\right|^{2}}{16 \pi m_{N}}\left[1-\left(1-r_{N}^{-2}\right) \log \left(1-r_{N}^{2}\right)\right],
$$

with $r_{N}=m_{N} / m_{H^{ \pm}}$. The effective low-energy nucleons form factors are obtained from chiral perturbation theory (please see [67-70] for a more detailed discussion). For the nuclear form factors the following values have been used: $f_{p}^{S u}=1.53 \times 10^{-2}, f_{p}^{S d}=$ $1.91 \times 10^{-2}, f_{p}^{S s}=4.47 \times 10^{-2}$, and $f_{p}^{S g}=1-\sum_{q=u, d, c} f_{p}^{S q}=0.9209$. The spin-independent cross section scales as $\lambda_{3}^{2}\left|y_{N}\right|^{4} / m_{N}^{2}$ modulo factors that depend on mass-splitting between the charged Higgs boson and the Majorana DM. To compute the relic abundance and Spin-independent Nucleus-DM elastic cross sections we use MadDM version 3.0 [71-73]. In figure 3, we show the relic density as a function of the DM mass $m_{N}$ for various fixed mass values of the charged Higgs bosons and $y_{N}$. We can see that the relic abundance of the $N$ avoid the over-abundance bound from the PLANCK experiment if one of the 




Figure 3. Dependence of the DM relic abundance as a function of its mass $m_{N}$ for various values of $y_{N}=\sqrt{y_{e}^{2}+y_{\mu}^{2}+y_{\tau}^{2}} \simeq y_{e}$ and the charged Higgs mass $m_{H^{ \pm}}$; the results are shown for $y_{N}=0.1$ (solid), $y_{N}=1$ (dashed), $y_{N}=2$ (dotted). The color codings for the charged Higgs masses are as follows: red lines correspond to $m_{H^{ \pm}}=200 \mathrm{GeV}$, green lines to $m_{H^{ \pm}}=500 \mathrm{GeV}$ and blue lines to $m_{H^{ \pm}}=1000 \mathrm{GeV}$. All the values are consistent with bounds from the Xenon1T searches of DM-Nucleon elastic scattering. All the calculations have been done for $\lambda_{3}=1$. The horizontal black solid line corresponds to the PLANCK measurement of relic density $\left(\Omega_{\text {Planck }} h^{2}=0.12\right)$.

following conditions are met: (i) relatively large Yukawa coupling $y_{N}>1$ and relatively light charged Higgs $m_{H^{ \pm}}<500 \mathrm{GeV}$ and (ii) for small mass-splittings, $\Delta<0.1$ which is the regime dominated by co-annihilations regardless the possible values of $y_{N}$. In the remaining part of this paper, we consider the following benchmark scenarios ${ }^{4}$

$$
100 \leqslant m_{H^{ \pm}} / \mathrm{GeV} \leqslant 1000, \quad 10 \leqslant m_{N} / \mathrm{GeV}<m_{H^{ \pm}}, \lambda_{3}=1, y_{N}=2 .
$$

A strong anti-correlation between $\Omega h^{2}$ and $\sigma_{\text {SI }}$ can be seen in figure 4 . The parameter space values produce a DM relic density $\Omega h^{2} \simeq \Omega_{\text {Planck }} h^{2}$ corresponding masses in the range $200-750 \mathrm{GeV}$ for which the corresponding spin-independent cross section is about $\simeq 10^{-48} \mathrm{~cm}^{2}$ which is below the XENON1T bound [3]. Moreover, as the mass splitting $\left(m_{H^{ \pm}}-m_{N}\right)$ gets smaller, the relic density decreases while still satisfying the XENON1T bound. It is also worth noting that the model is unconstrained from the current indirect detection experiments due to the fact that $s$-channel annihilation channels are loop induced and mediated by the SM Higgs boson, with the predicted annihilation cross sections $\langle\sigma v\rangle \simeq$ $10^{-37} \mathrm{~cm}^{3} / s$ that is orders of magnitude smaller than the FERMI-LAT bounds [74].

Before closing this section, we discuss briefly the impact of changing the values of $\lambda_{2}$ and $\lambda_{3}$ on our results. First, we note that the variations of $\lambda_{2}$ do not change the results

\footnotetext{
${ }^{4}$ The collider implications of the scenario with $y_{N}<0.1$ in which the DM relic abundance is dominated by the co-annihilation process will be studied in a future work.
} 


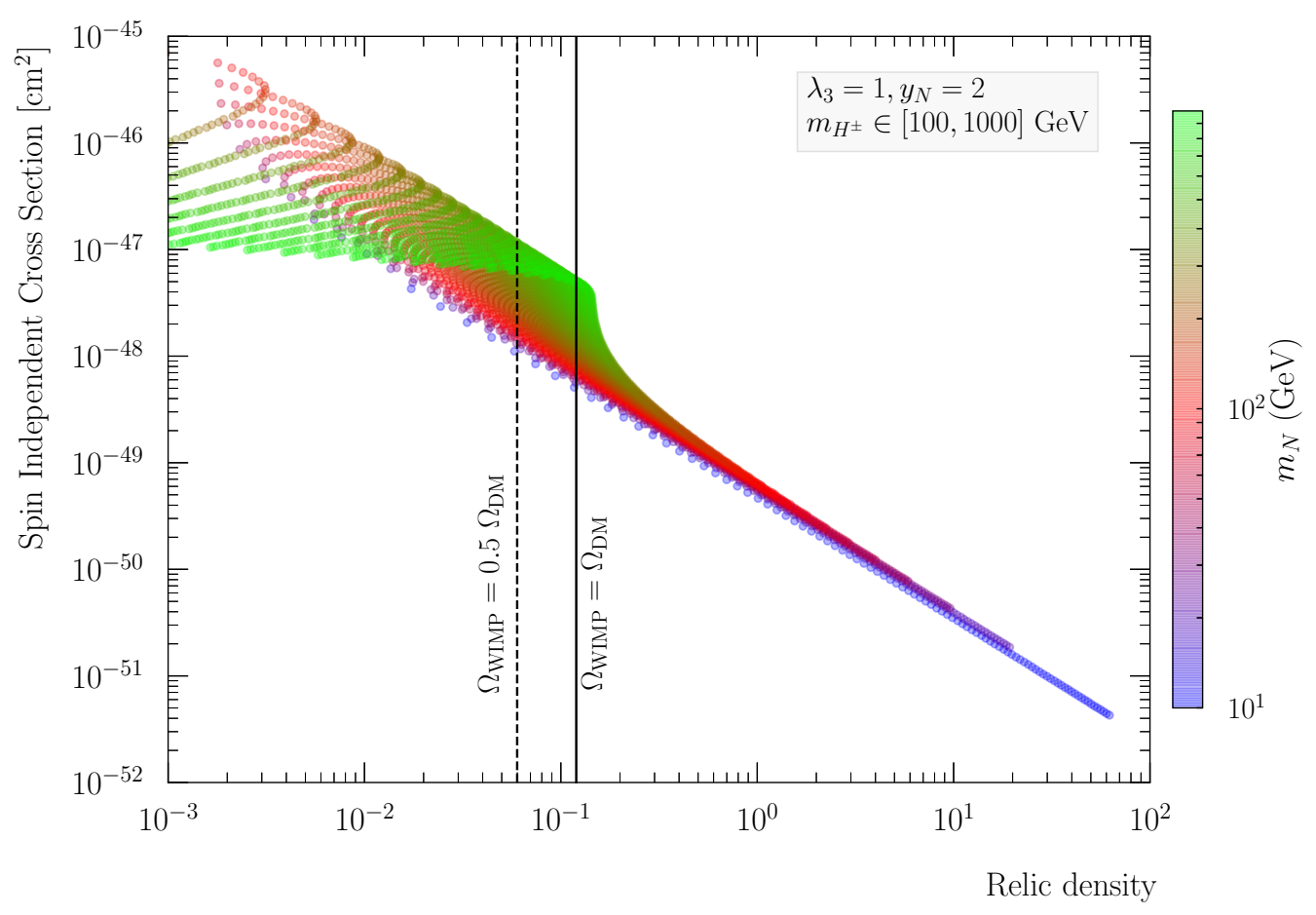

Figure 4. Correlation between the relic density and the spin-independent direct detection cross section. The palette shows the value of the DM mass. The two vertical lines correspond to $\Omega_{N} h^{2}=$ $\Omega_{\text {Planck }} h^{2}$ (solid) and $\Omega_{N} h^{2}=0.5 \Omega_{\text {Planck }} h^{2}$ (dashed).

of the analysis especially for the considered benchmarks in eq. (5.9) in which $\lambda_{3}=1$. The main net effect of changing $\lambda_{2}$ is constraining the parameter space through the theoretical constraints. If $\lambda_{2}$ is large enough (say greater than 2) the allowed regions of $\lambda_{3}$ become very restricted. However, this does not affect our analysis as $\lambda_{3}$ around one is always permitted by theoretical constraints. It is true that modifications of especially $\lambda_{3}$ may change the results of our study but this change can be accommodated by a simple scaling of the resulting cross sections as $\sigma_{e^{+} e^{-} \rightarrow H N N} \propto \lambda_{3}^{2}, \Gamma_{H \rightarrow \text { invisible }} \propto \lambda_{3}^{2}$ and $\sigma_{\mathrm{SI}} \propto \lambda_{3}^{2}$. Therefore, if one increases $\lambda_{3}$, the expected constraints from mono-Higgs searches at the

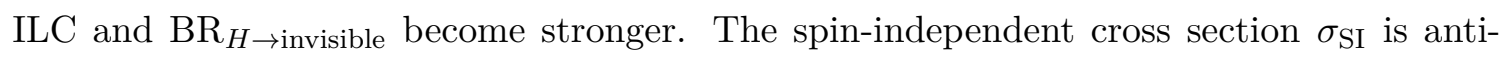
correlated with the relic density, and therefore regions in the parameter space enhancing $\sigma_{\mathrm{SI}}$ would correspond to $\Omega_{\mathrm{DM}} h^{2} / \Omega_{\text {Planck }} h^{2} \ll 1$. On the other hand, co-annihilations become more active for larger $\lambda_{3}$ and small mass splittings. Hence, The present bounds from $\sigma_{\mathrm{SI}}$ would not affect the model parameter space since the predicted $\sigma_{\mathrm{SI}}$ should be re-scaled by $\xi \equiv \Omega_{\mathrm{DM}} h^{2} / \Omega_{\text {Planck }} h^{2}$.

\section{Prospects at the International Linear Collider}

Our model gives rise to several signatures at the future International Linear Collider (ILC) that is expected to run at center-of-mass energies of $250,350,500$ and $1000 \mathrm{GeV}$ [75] and that will include the collision of electron-electron beams [76,77]. The advantage of the latter option is that it can be used efficiently to probe lepton-violating processes due to the small 


\begin{tabular}{|c|ccc|ccc|}
\hline$\sqrt{s}[\mathrm{GeV}]$ & \multicolumn{3}{|c|}{500} & \multicolumn{3}{|c|}{1000} \\
\hline$\left[P_{e^{-}}, P_{e^{+}}\right]$ & {$[0,0]$} & {$[+0.8,-0.3]$} & {$[-0.8,+0.3]$} & {$[0,0]$} & {$[+0.8,-0.2]$} & {$[-0.8,+0.2]$} \\
\hline$W^{+} W^{-}$ & 7.19 & 1.06 & 16.77 & 2.68 & 0.44 & 5.79 \\
$Z Z$ & 0.42 & 0.32 & 0.71 & 0.15 & 0.11 & 0.24 \\
$t \bar{t}$ & 0.55 & 0.44 & 0.92 & 0.16 & 0.13 & 0.25 \\
$H \nu \bar{\nu}_{e}$ & $7.76 \times 10^{-2}$ & $1.14 \times 10^{-2}$ & 0.18 & 0.21 & $3.44 \times 10^{-2}$ & 0.45 \\
$H Z$ & $5.72 \times 10^{-2}$ & $5.72 \times 10^{-2}$ & $8.47 \times 10^{-2}$ & $1.28 \times 10^{-2}$ & $1.21 \times 10^{-2}$ & $1.77 \times 10^{-2}$ \\
\hline
\end{tabular}

Table 1. Production cross of the major background processes to the mono-Higgs channel in $e^{+} e^{-}$ collisions at $\sqrt{s}=500 \mathrm{GeV}$ and $\sqrt{s}=1000 \mathrm{GeV}$. The cross sections, given in picobarn, are calculated at the Leading-Order ( $\mathrm{LO})$ in perturbation theory.

background contributions. At electron-positron colliders, there are several processes that can be used to probe the model; $(i): e^{+} e^{-} \rightarrow N N Z$ which yields a signature dubbed mono$Z$, (ii): $e^{+} e^{-} \rightarrow N N \gamma$ which yields one photon in association with large missing energy, (iii): $e^{+} e^{-} \rightarrow N N H$ which yields a variety of final states depending on the decay products of the SM Higgs boson, (iv): $e^{+} e^{-} \rightarrow H^{ \pm} H^{\mp}$ which yields two opposite-sign charged leptons plus missing energy, and $(v): e^{-} e^{-} \rightarrow H^{-} H^{-}$which yields two same-sign charged leptons in association with large missing energy. In addition to these tree-level direct production processes, our model can be probed indirectly through radiative corrections of the rates of the Higgs-strahlung and double Higgs-strahlung at lepton-colliders. For instance, the rate of $H Z$ production at lepton colliders gets three major contributions: (i) triangle corrections to the initial $e^{+} e^{-} Z$ vertex with the exchange of the DM and the charged Higgs boson, (ii) box corrections with the exchange of the DM and the charged Higgs bosons, and (iii) triangle corrections to the final $\gamma^{*} / Z Z H$ vertex with the exchange of charged Higgs bosons only. However, only the last contribution has been explicitly computed in the framework of the inert Higgs doublet model which involves two extra scalar inert scalars $H^{0} / A^{0}$ as well. At $\sqrt{s}=250 \mathrm{GeV}$, it is found that these corrections are at the percent-level [78]. A full analysis of this process within the framework of our model is beyond the scope of this work.

We consider two signal processes; mono-Higgs process in $e^{+} e^{-}$collisions at $\sqrt{s}=500$, $1000 \mathrm{GeV}$, and the same-sign charged Higgs pair production in $e^{-} e^{-}$collisions at $1000 \mathrm{GeV} .^{5}$

For the mono-Higgs process, we consider the $H \rightarrow b \bar{b}$ decay channel of the SM Higgs boson for which the major backgrounds are $H(\rightarrow b \bar{b}) Z(\rightarrow \nu \bar{\nu}), H(\rightarrow b \bar{b}) \nu_{e} \bar{\nu}_{e}, W^{+} W^{-}, Z Z$, and $t \bar{t}$ (the corresponding cross sections are given in table 1). Signal and backgrounds processes are generated using Madgraph5_aMC@NLO [49] and passed to Pythia8 [55] to add parton showering, hadronisation, and hadron decays. We employ Delphes [87] to account for detector angularity, jet smearing, and particle resolutions. Jets are clustered according to the anti- $k_{T}$ algorithm [57] with $R=0.4$ using Fast Jet [56]. In figure 5, we present the differential cross sections for some key variables that we used in the signal-to-backgrounds optimisation for signal process and the main backgrounds. In that figure, we show the

\footnotetext{
${ }^{5}$ Other studies for DM production at lepton colliders have been performed in [79-86] for different DM models.
} 


\begin{tabular}{|ccccc|}
\hline Cut & $W^{+} W^{-} / Z Z$ & $t \bar{t}$ & $H \nu_{e} \bar{\nu}_{e}$ & $H Z$ \\
\hline Initial events & $2 \times 10^{6}(100 \%)$ & $10^{6}(100 \%)$ & $10^{6}(100 \%)$ & $10^{6}(100 \%)$ \\
Lepton veto & $476982(23.84 \%)$ & $29449(2.94 \%)$ & $486875(48.68 \%)$ & $171353(17.13 \%)$ \\
$n_{b}=2$ & $17885(0.89 \%)$ & $5275(0.52 \%)$ & $133594(13.35 \%)$ & $40031(4.00 \%)$ \\
$p_{T}^{b \bar{b}}>50 \mathrm{GeV}$ & $10025(0.50 \%)$ & $3572(0.36 \%)$ & $84701(8.47 \%)$ & $34975(3.49 \%)$ \\
$E_{T}^{\text {miss }}>50 \mathrm{GeV}$ & $9559(0.48 \%)$ & $3175(0.32 \%)$ & $83846(8.38 \%)$ & $34286(3.43 \%)$ \\
Signal region & $2\left(2 \times 10^{-6}\right)$ & $372(0.037 \%)$ & $18339(1.83 \%)$ & $733(0.073 \%)$ \\
\hline Initial events & $2 \times 10^{6}(100 \%)$ & $10^{6}(100 \%)$ & $10^{6}(100 \%)$ & $10^{6}(100 \%)$ \\
Lepton veto & $432342(21.61 \%)$ & $15637(1.56 \%)$ & $493589(49.35 \%)$ & $203586(20.35 \%)$ \\
$n_{b}=2$ & $15464(0.77 \%)$ & $3378(0.34 \%)$ & $138187(13.82 \%)$ & $48596(4.85 \%)$ \\
$p_{T}^{b \bar{b}}>50 \mathrm{GeV}$ & $9973(0.49 \%)$ & $2713(0.27 \%)$ & $102302(10.23 \%)$ & $42249(4.22 \%)$ \\
$E_{T}^{\text {miss }}>50 \mathrm{GeV}$ & $8980(0.45 \%)$ & $2565(0.25 \%)$ & $101669(10.16 \%)$ & $42032(4.20 \%)$ \\
Signal region & $1\left(10^{-6}\right)$ & $26(0.0026 \%)$ & $3541(0.35 \%)$ & $631(0.063 \%)$ \\
\hline
\end{tabular}

Table 2. The cutflow table for various background contributions after each selection step. We show the cases $\sqrt{s}=500 \mathrm{GeV}$ (upper panels) and $\sqrt{s}=1000 \mathrm{GeV}$ (lower panels). The number inside the parentheses show the efficiency after the selection step $i$ which is defined as $\epsilon=n_{i} / n_{0}$ with $n_{0}$ is the initial number of events.

transverse momentum of the $b \bar{b}$ system $\left(p_{T}^{b \bar{b}}\right)$, the missing transverse energy $\left(E_{T}^{\text {miss }}\right)$, the invariant mass of the $b \bar{b}$ system and of the reconstructed invisible momentum.

For the event selection, we start by considering events that pass the following preselection criteria

- Lepton veto: no isolated lepton (electron or muon) with $p_{T}^{\ell}>15 \mathrm{GeV}$ and $\left|\eta^{\ell}\right|<2.5$.

- Exactly two $b$-tagged jets with $p_{T}^{b}>30 \mathrm{GeV}$ and $\left|\eta^{b}\right|<2.5$.

Furthermore, the two $b$-tagged jets are used to form Higgs boson candidate whose transverse momentum is required to be larger than $50 \mathrm{GeV}$. The invariant mass of the invisible system is related to the energy of the Higgs boson candidate by the following relation

$$
M_{\mathrm{inv}}^{2}=s-2 \sqrt{s} E_{b \bar{b}}+M_{b \bar{b}}^{2},
$$

with $s$ being the center-of-mass energy, and $E_{b \bar{b}}\left(M_{b \bar{b}}\right)$ is the energy (the invariant mass) of the $b \bar{b}$ system. We define the signal region by: (i) the invariant mass of the $b \bar{b}$ system is required to satisfy $\left|M_{b \bar{b}}-m_{H}\right|<10 \mathrm{GeV}$, and (ii) the invariant mass of the invisible system to satisfy $200 \mathrm{GeV}<M_{\text {inv }}<800 \mathrm{GeV}$. For completeness, we show the cutflow for the different background contributions in table 2. We note that the selection efficiency for both the signal as well as the backgrounds do not depend drastically on the polarisation setup of the incoming $e^{+} / e^{-}$beams, and the efficiency of the signal process, in the signal region, varies in the range $2 \%-3 \%$.

The charged Higgs pair production in the electron-electron option at the ILC is certainly very interesting. The reasons are two-fold; $(i)$ the signal is enhanced for large DM 

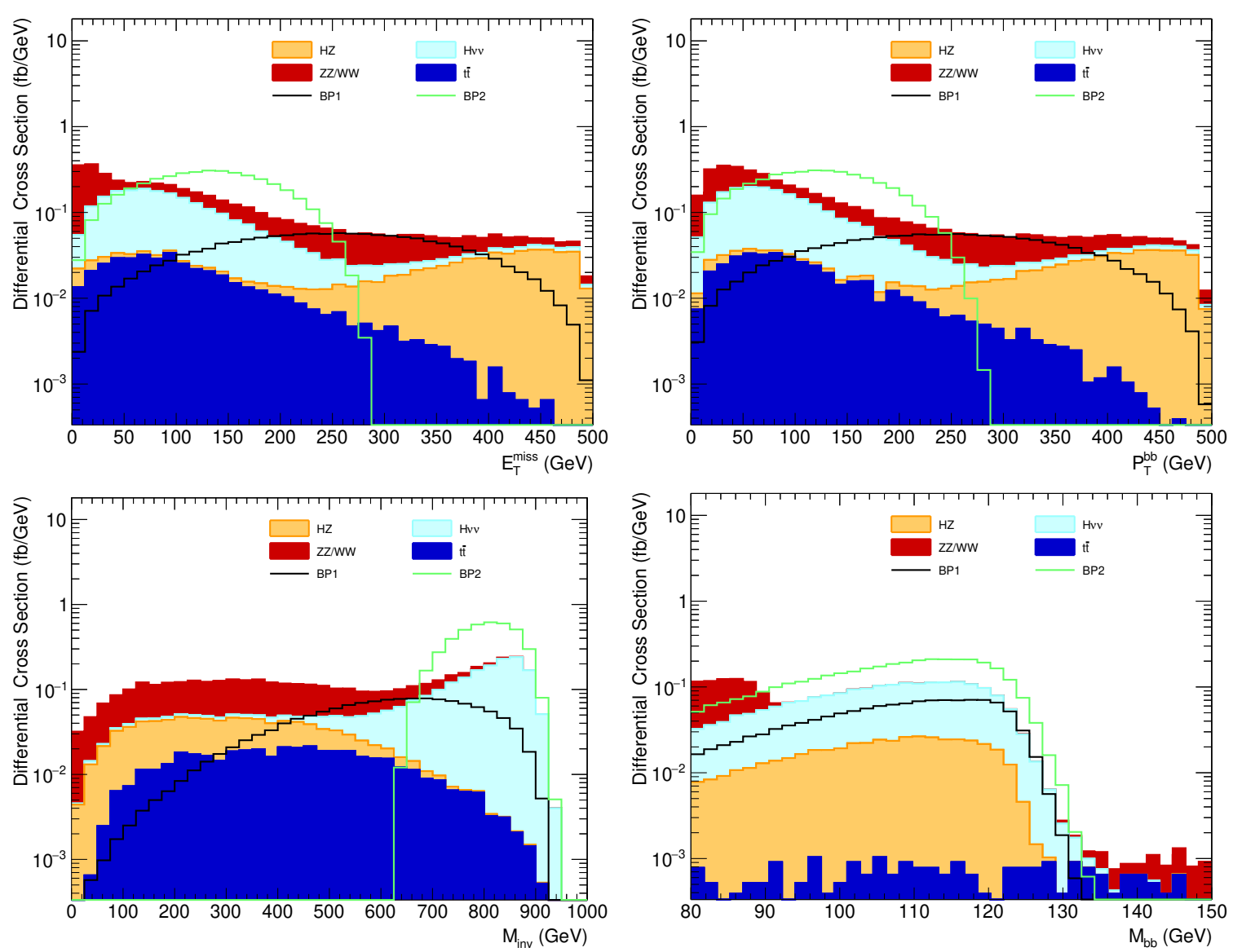

Figure 5. Differential cross sections for some selected observables used in the signal-to-background discrimination in $e^{+} e^{-} \rightarrow H+E_{T}^{\text {miss }}$ process at $\sqrt{s}=1000 \mathrm{GeV}$, and assuming $\left[P_{e^{-}}, P_{e^{+}}\right]=$ $[+80 \%,-20 \%]$. From top left to bottom right, we display the missing transverse energy $\left(E_{T}^{\text {miss }}\right)$, the transverse momentum of the $b \bar{b}$ system $\left(p_{T}^{b \bar{b}}\right)$, the invisible invariant mass $\left(M_{\mathrm{inv}}\right)$, and the invariant mass of the $b \bar{b}$ system $\left(M_{b \bar{b}}\right)$. We show the distributions for $H Z$ (orange), diboson $(Z Z / W W)$, $H \nu_{e} \bar{\nu}_{e}$ (cyan) and $t \bar{t}$ (blue). For comparison, we show the differential cross sections for two signal benchmark points: BP1 corresponding to $m_{H^{ \pm}}=1000 \mathrm{GeV}$, and $m_{N}=20 \mathrm{GeV}$ (black line) and BP2 corresponding to $m_{H^{ \pm}}=460 \mathrm{GeV}$, and $m_{N}=320 \mathrm{GeV}$ (green line). The distributions are shown after the first two basic selections.

masses due to the Majorana nature of the DM, and (ii) the associated backgrounds are extremely suppressed since the SM processes conserve lepton number. Moreover, contrary to the mono-Higgs process, charged Higgs pair production can probe the whole parameter space of the model that can allowed by the collider energy. The cross section for the signal behaves as [88]

$$
\sigma_{e^{-} e^{-} \rightarrow H^{-} H^{-}} \propto m_{N}^{2} y_{e}^{4}
$$

Considering the decay of the charged Higgs boson into $e^{-} N$, which is the dominant one due to our choice of $y_{\ell}$ in section 3, the main backgrounds are $W^{-} W^{-} \nu_{e} \nu_{e}$ and $Z e^{-} e^{-}$ whose corresponding cross sections are $21.53 \mathrm{fb}$ and $98.1 \mathrm{fb}$ respectively. In figure 6 , we display the cross section for the signal process for $y_{N}=2$ (left panel). For comparison, 

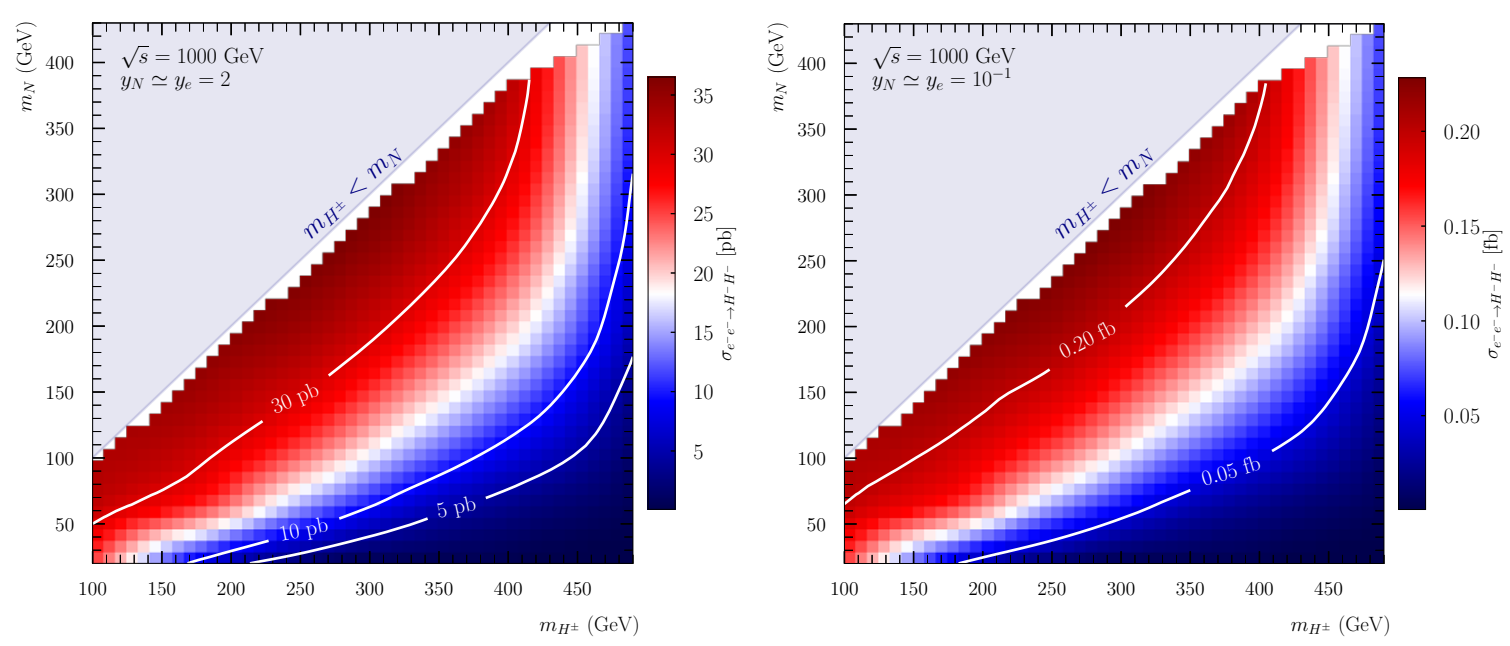

Figure 6. Production cross sections for same-sign charged Higgs pair production in $e^{-} e^{-}$collisions at $\sqrt{s}=1000 \mathrm{GeV}$ for $y_{N} \simeq y_{e}=2$ (left panel) and $y_{N} \simeq y_{e}=10^{-1}$ (right panel).

we show in the right panel of figure 6 , the cross section for $y_{N}=0.1$. We can see that for $y_{N}=2$, the signal cross section is orders of magnitude higher than the background ones; for $m_{H^{ \pm}}=250 \mathrm{GeV}$ we have $\sigma_{e^{-} e^{-} \rightarrow H^{-} H^{-}}=5.46(32.94) \mathrm{pb}$ for $m_{N}=30$ (180) GeV. Given the large signal-to-background ratios, we only apply some minor selections on the decay products of the charged Higgs; i.e. we select events that contain exactly two same-sign electrons with $p_{T}^{e}>20 \mathrm{GeV}$, and $\left|\eta^{e}\right|<2.5$.

To obtain the expected exclusions on the parameter space from mono-Higgs process and the same-sign charged Higgs pair production at the ILC, we compute the $\mathrm{CL}_{s}$ following the lines of section B. Since the number of observed events are not known yet, we assume it is equal to the expected number of backgrounds obtained from $\mathrm{MC}$ simulations at LO. On the other hand, we assume a flat $10 \%$ on the expected background events. A point in the space paramater of the model is expected to be excluded at the $95 \%$ CL if the corresponding $\mathrm{CL}_{s}$ is smaller than 0.05. In figure 7, we show the future sensitivity from slepton searches obtained from extrapolation of the exisiting LHC limits (green), mono-Higgs process (blue and red) and same-sign charged Higgs production (gray) on the charged Higgs mass and the mass of DM for $y_{N}=2$ and $\lambda_{3}=1$. The following observations can be made:

- The processes at hadron colliders cannot probe the region of the parameter space with small mass-splittings; i.e. $m_{H^{ \pm}}-m_{N}<50 \mathrm{GeV}$. The reason is that the charged leptons produced from charged Higgs production are relatively soft and do not pass the threshold of the lepton selection in addition to the strong refinement from transverse missing energy criteria. On the other hand, the ATLAS search (which was used in obtaining thse limits) relies on the stransverse mass $\left(M_{T 2}\right)$ which has usually an end-point at the mass of the decaying charged Higgs bosons. Therefore, the inclusive signal regions defined by ATLAS cannot probe light charged Higgs bosons in our scenario. 


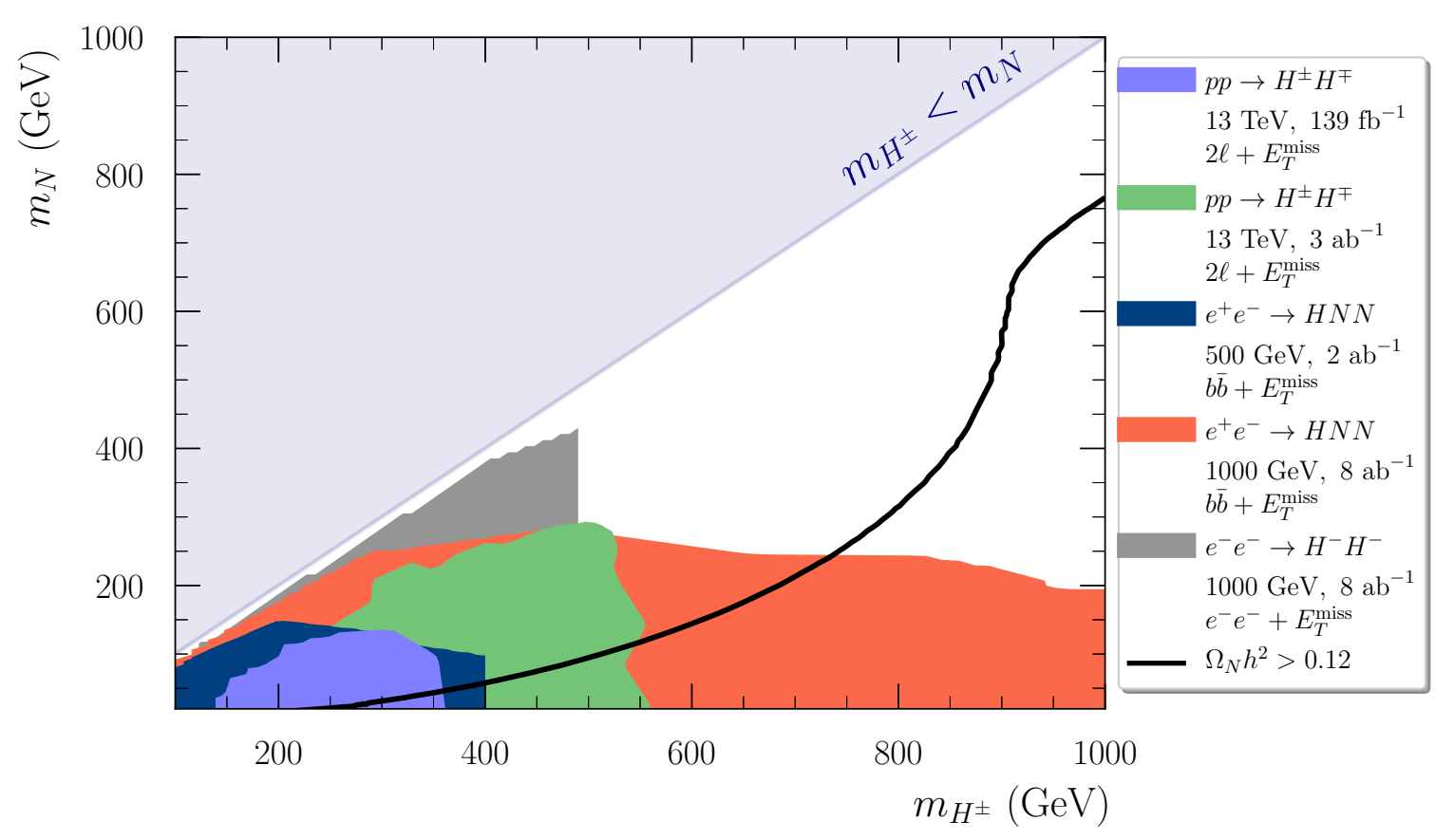

Figure 7. A summary of the impact of the present and future colliders on the parameter space of our model projected on the plane of the charged Higgs mass $\left(m_{H^{ \pm}}\right)$and the mass of DM $\left(m_{N}\right)$. The shaded purple area shows the excluded parameter space from the re-interpretation of sleptons searches at the LHC with $\mathcal{L}=139 \mathrm{fb}^{-1}$. The green shaded region shows the extrapolations of the LHC limits to high luminosities $\left(\mathcal{L}=3000 \mathrm{fb}^{-1}\right)$. The expected exclusions from mono-Higgs searches at the ILC at both $\sqrt{s}=500 \mathrm{GeV}$ and $\sqrt{s}=1000 \mathrm{fb}^{-1}$ are shown in blue and red shaded areas respectively. Finally, the expected limits from searches of same-sign charged Higgs pair production at $\sqrt{s}=1000 \mathrm{GeV}$ are shown as gray contour. We show the contour satisfying $\Omega_{N} h^{2}=0.12$ as black line (the area below which is excluded). All these results were obtained assuming $y_{N} \simeq y_{e}=2$ and $\lambda_{3}=1$. The bounds from Xenon1T experiment are fulfilled.

- The mono-Higgs process can be used to probe DM masses up to $200 \mathrm{GeV}$ independently of the charged Higgs boson mass. However, this process is strongly dependent on both $\lambda_{3}$ and $y_{N}$. Decreasing the values of those parameters will reduce the expected event yields and, therefore, weakens the limits.

- The rate of the same-sign charged Higgs pair production grows quadratically with the mass of the Majorana DM, and the associated backgrounds are essentially negligible. Therefore, this process can probe all the regions of the parameter space allowed by the collider energy provided that $y_{N}$ is of order unity or larger.

\section{Conclusions}

In summary, we studied an interesting scenario which may provide DM candidate and at the same time an explanation for the absence of DM signals in both direct detection experiments and at colliders. In this framework, we minimally extended the SM by two gauge singlets: a charged scalar and a Majorana singlet fermion. First, we studied the impact 
of different theoretical and experimental constraints on the model parameter space, and showed that it can be severely constrained by limits from lepton-flavor violating decays at present experiments which in turn would suppress the magnitude of the couplings of the DM candidate to the visible sector. On the other hand, Higgs boson invisible decays, if observed in future experiments, would further restricts the range of the couplings for relatively light $\mathrm{DM} ; m_{\mathrm{DM}}<m_{H} / 2$. Then, using simple correlations between the relic abundance and the spin-independent cross sections, we showed that DM masses consistent with the PLANCK observations are still allowed by the XENON1T bounds, and well above the neutrino-floor. Besides, we found that this model can be probed at lepton colliders using the mono-Higgs process and the same-sign charged Higgs pair production. This simple model provides a one-to-one correspondence between its parameters and the predicted values for physical observables. For instance, the mono-Higgs signature of the model can be used to probe both $m_{N}$ and the product of $\lambda_{3}$ and $y_{N}$. An inference on these parameters from the measurement of the rate of this process will restrict the dependence of the predictions for direct detection experiments on them - we note that $\sigma_{\mathrm{SI}}$ involves an additional dependence on $m_{H^{ \pm}}$. The same-sign charged Higgs boson pair production is anti-correlated to the relic density and is proportional to $y_{N}^{4} m_{N}^{2}$. Therefore, for the set of parameters $\left\{\lambda_{3}, y_{N}, m_{H^{ \pm}}, m_{N}\right\}$ there are four corresponding observables, i.e. $\left\{\sigma_{e^{+} e^{-} \rightarrow H N N}, \sigma_{e^{-} e^{-} \rightarrow H^{-} H^{-}}, \sigma_{\mathrm{SI}}, \Omega h^{2}\right\}$. Important steps are yet to be made regarding the probes of these scenarios using one of the many developed methods to study the characteristics of DM at colliders. One can also obtain the interaction (2.2) from a more UV complete model. For instance, this can be realized by embedding the SM into SU(5) gauge group with the matter fields in $\mathbf{1 0}$ and $\overline{\mathbf{5}}$ representation and the charged singlet belongs to the $\mathbf{1 0}_{H}$ representation, while the right handed neutrino belongs to the singlet representation, i.e. $\mathcal{L}_{\text {int }}=g_{\alpha \beta} \overline{\mathbf{1 0}}_{\alpha} \otimes \mathbf{1 0}_{H} \otimes \mathbf{1}_{N_{\beta}} \supset g_{\alpha \beta} \ell_{R \alpha}^{T} C N_{\beta} S^{+}$. Another possibility is the flipped-SU $(5) \times \mathrm{U}(1)_{X}$ grand-unified theory, where the right handed lepton field is singlet of $\mathrm{SU}(5)$, and the right handed neutrino is a member of the $\mathbf{1 0}_{\alpha}$ representation. In this case, the interaction (2.2) can be obtained from the following effective Lagrangian $\mathcal{L}_{\text {int }}=\frac{h_{\alpha \beta}}{\Lambda} \overline{\mathbf{1 0}}_{\alpha} \otimes \overline{\mathbf{1}}_{\beta} \otimes \mathbf{1 0}_{H} \otimes \mathbf{1}_{S}+$ h.c. $\supset \frac{h_{\alpha \beta}\left\langle\mathbf{1 0}_{H}\right\rangle}{\Lambda} N^{T} C \ell_{R} S^{-}$. The embedding of our model into a grand-unified theory is certainly an interesting question to pursue which we report on for a future study [89].

\section{Acknowledgments}

Most of the plots in this paper were made using Matplotlib [90]. Numerical and Statistical calculations were made using NumPy and SciPy [91, 92]. AJ would like to thank the CERN Theoretical Physics Department for its hospitality where a part of this work has been done. AJ would like to thank Jack Araz and Benjamin Fuks for providing the necessary MadAnalysis routines corresponding to the implementation of the ATLAS-SUSY-18-032 search which we used in this work to obtain the LHC constraints on the model parameter space. The work of AJ is supported by the National Research Foundation of Korea, Grant No. NRF-2019R1A2C1009419. 




(a)

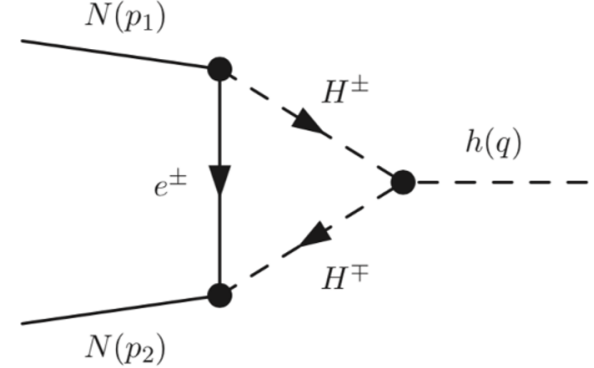

(b)

Figure 8. One-loop Feynman diagram for the $H(q) \rightarrow N\left(p_{1}\right) N\left(p_{2}\right)$ process which gives rise to the effective $\tilde{y}_{H N N}$ coupling.

\section{A Computation of the effective $\tilde{y}_{H N N}$ coupling}

In this section, we show the detailed calculation of the effective $\tilde{y}_{H N N}$ coupling. To do so, we consider the process

$$
H(q) \longleftrightarrow N\left(p_{1}\right)+\bar{N}\left(p_{2}\right)
$$

where in eq. (A.1), $p_{1}$ and $p_{2}$ are the four-momenta of the right handed Neutrinos satisfying the on-shell constraint $p_{1}^{2}=p_{2}^{2}=m_{N}^{2}$ and $q$ is the four-momentum of the Higgs boson. An example of Feynman diagram is displayed in figure 8.

The corresponding Feynman amplitude can be written as

$$
\begin{aligned}
\mathcal{M} & =\sum_{\ell=e, \mu, \tau} \int \frac{\mathrm{d}^{4} k}{(2 \pi)^{4}} \frac{\bar{v}\left(p_{2}\right)\left(-i y_{\ell}^{*} P_{R}\right) i\left(\not k+m_{\ell}\right)\left(-i y_{\ell} P_{L}\right)\left(-i \lambda_{3} v\right) u\left(p_{1}\right)}{\left(k^{2}-m_{\ell}^{2}\right)\left(\left(p_{2}+k\right)^{2}-m_{H^{ \pm}}^{2}\right)\left(\left(k-p_{1}\right)^{2}-m_{H^{ \pm}}^{2}\right)}+(R \longleftrightarrow L), \\
& =\sum_{\ell=e, \mu, \tau} \lambda_{3} v\left|y_{\ell}\right|^{2} \int \frac{\mathrm{d}^{4} k}{(2 \pi)^{4}} \frac{\bar{v}\left(p_{2}\right) P_{R} \not k P_{L} u\left(p_{1}\right)}{k^{2}\left(\left(k+p_{2}\right)^{2}-m_{H^{ \pm}}^{2}\right)\left(\left(k-p_{1}\right)^{2}-m_{H^{ \pm}}^{2}\right)}+(R \longleftrightarrow L) .
\end{aligned}
$$

In the second line, we have ignored the mass of the charged lepton in both the numerator and the denominator. After redefining the integration variables, and making few mathematical manipulations, we get:

$$
\mathcal{M}=\lambda_{3} v\left|y_{N}\right|^{2}\left(\bar{v}\left(p_{2}\right) P_{R} \mathcal{I}_{(I)}^{\mu} \gamma_{\mu} P_{L} u\left(p_{1}\right)-\bar{v}\left(p_{2}\right) P_{R} \not p_{2} \mathcal{I}_{(I I)} P_{L} u\left(p_{1}\right)\right)+\left(\gamma_{5} \rightarrow-\gamma_{5}\right)
$$

where we have used $\left|y_{N}\right|^{2}=\sum_{\ell=e, \mu, \tau}\left|y_{\ell}\right|^{2}$. The integrals we need to evaluate are

$$
\begin{aligned}
\mathcal{I}_{(I)} & =\int \frac{\mathrm{d}^{4} k}{(2 \pi)^{4}} \frac{k^{\mu}}{\left(k-p_{2}\right)^{2}\left(k^{2}-m_{H^{ \pm}}^{2}\right)\left(\left(k-p_{1}-p_{2}\right)^{2}-m_{H^{ \pm}}^{2}\right)} \\
\mathcal{I}_{(I I)} & =\int \frac{\mathrm{d}^{4} k}{(2 \pi)^{4}} \frac{1}{k^{2}\left(\left(k+p_{2}\right)^{2}-m_{H^{ \pm}}^{2}\right)\left(\left(k-p_{1}\right)^{2}-m_{H^{ \pm}}^{2}\right)} .
\end{aligned}
$$

Evaluating these integrals using dimensional regularization, one easily gets

$$
\mathcal{I}_{(I)}^{\mu}=\frac{i}{16 \pi^{2}}\left(p_{2}^{\mu} C_{2}+\left(p_{1}^{\mu}+p_{2}^{\mu}\right) C_{1}\right), \quad \mathcal{I}_{(I I)}=\frac{-i}{16 \pi^{2}} C_{0},
$$


with

$$
C_{i} \equiv C_{i}\left(m_{N}^{2}, q^{2}, m_{N}^{2}, 0, m_{H^{ \pm}}^{2}, m_{H^{ \pm}}^{2}\right), i=0,1,2 .
$$

are the three-point Passarino-Veltman functions [40]. Inserting the expressions of the integrals into eq. (A.3), and using Dirac equations, we find:

$$
\mathcal{M}=\frac{\lambda_{3} v\left|y_{N}\right|^{2} m_{N}}{16 \pi^{2}} \bar{v}\left(p_{2}\right) u\left(p_{1}\right)\left(C_{0}+C_{2}\right) .
$$

To get the effective $\tilde{y}_{H N N}$ coupling, we estimate the amplitude in equation (A.6) for $q^{2} \simeq 0$. The Leading terms in the expansions of the Passarino-Veltman functions near $q^{2} \simeq 0$ are given by ${ }^{6}$

$$
\begin{aligned}
C_{0} & \simeq \frac{1}{m_{N}^{2}} \log \left(\frac{m_{H^{ \pm}}^{2}-m_{N}^{2}}{m_{H^{ \pm}}^{2}}\right)+\mathcal{O}\left(q^{2}\right), \\
C_{2} & \simeq \frac{-1}{m_{N}^{4}}\left[m_{N}^{2}+m_{H^{ \pm}}^{2} \log \left(\frac{m_{H^{ \pm}}^{2}-m_{N}^{2}}{m_{H^{ \pm}}^{2}}\right)\right]+\mathcal{O}\left(q^{2}\right) .
\end{aligned}
$$

Therefore, we find the expression of the $\tilde{y}_{H N N}$ coupling at $q^{2} \simeq 0$

$$
\tilde{y}_{H N N}=\frac{-\lambda_{3} v\left|y_{N}\right|^{2}}{16 \pi^{2} m_{N}^{3}}\left[m_{N}^{2}+\left(m_{H^{ \pm}}^{2}-m_{N}^{2}\right) \log \left(1-\frac{m_{N}^{2}}{m_{H^{ \pm}}^{2}}\right)\right]+\mathcal{O}\left(q^{2}\right),
$$

which is in excellent agreement with the results of [47, 94].

\section{B LHC limits: Signal Regions (SR) and the statistical treatment}

In this section, we describe some details about the statistical setup, the exclusion bounds for all the considered inclusive signal regions as well as the method used to obtain the limit extrapolation up to higher expected luminosities. The ATLAS collaboration has tackled such an analysis by defining four inclusive signal regions for each jet bin category, based on the $M_{T 2}$ interval splitting variable. The summary of the expected background and observed event numbers is given in table 3 with different signal region definitions.

The exclusion limits on the parameter space based on the $\mathrm{CL}_{s}$ prescription are obtained using MadAnalysis. Initially, the signal event number passing the selection of a given signal region can be estimated as follows:

$$
N_{\mathrm{s}}=\epsilon_{\mathrm{s}} \mathcal{L} \sigma
$$

where $\mathcal{L}$ represents the recorded integrated luminosity $\left(139 \mathrm{fb}^{-1}\right), \epsilon_{\mathrm{s}}$ is the cumulative efficiency within the $\mathrm{SR}$, and $\sigma=\sigma\left(p p \rightarrow H^{+} H^{-} j j\right) \times \mathrm{BR}_{H^{ \pm} \rightarrow \ell^{ \pm} N}^{2}$ is the merged production cross section of the Charged Higgs pairs. For each defined signal region, the observed event yield $\left(n_{\mathrm{obs}}\right.$ ), the fitted background yields $\left(n_{\mathrm{b}}\right)$, and the uncertainty on the background predictions $\left(\Delta n_{\mathrm{b}}\right)$ are provided (see table 3 for details). A large number of toy experiments, denoted by $N_{\text {toys }}$, is generated where in each round the $p_{\mathrm{b}}$ and $p_{\mathrm{b}+\mathrm{s}}$ probabilities are calculated following the steps:

\footnotetext{
${ }^{6}$ We have checked the correctness of our calculations by comparing the analytical expansions of the Passarino-Veltman function near $q^{0} \simeq 0$ with the output we got using Package-X [93].
} 


\begin{tabular}{|ccccc|}
\hline $\begin{array}{c}\text { Region } \\
M_{T 2}(\mathrm{GeV})\end{array}$ & $\begin{array}{c}\text { SR-SF-0J } \\
\in[100, \infty)\end{array}$ & $\begin{array}{c}\text { SR-SF-0J } \\
\in[160, \infty)\end{array}$ & $\begin{array}{c}\text { SR-SF-0J } \\
\in[100,120)\end{array}$ & $\begin{array}{c}\text { SR-SF-0J } \\
\in[120,160)\end{array}$ \\
\hline Observed events & 147 & 37 & 53 & 57 \\
\hline Fitted backgrounds & $144 \pm 12$ & $37.3 \pm 3.0$ & $56 \pm 6$ & $51 \pm 5$ \\
\hline$m_{H^{ \pm}}, m_{N}=(200,10) \mathrm{GeV}$ & 109.6 & 36.7 & 23.9 & 48.9 \\
$m_{H^{ \pm}}, m_{N}=(200,142) \mathrm{GeV}$ & 1.7 & 0.0 & 1.5 & 0.2 \\
\hline Region & SR-SF-1J & SR-SF-1J & SR-SF-1J & SR-SF-1J \\
$M_{T 2}(\mathrm{GeV})$ & $\in[100, \infty)$ & $\in[160, \infty)$ & $\in[100,120)$ & $\in[120,160)$ \\
\hline Observed events & 120 & 29 & 55 & 36 \\
\hline Fitted backgrounds & $124 \pm 12$ & $36 \pm 5$ & $48 \pm 8$ & $40 \pm 4$ \\
\hline$m_{H^{ \pm}}, m_{N}=(200,10) \mathrm{GeV}$ & 53.7 & 19.1 & 11.3 & 23.4 \\
$m_{H^{ \pm}}, m_{N}=(200,142) \mathrm{GeV}$ & 1.3 & 0.0 & 1.2 & 0.1 \\
\hline
\end{tabular}

Table 3. Observed and expected background event yields for the 'same flavor' (SF) inclusive signal regions SRs (those numbers are taken from [48]). For reference, we show the number of events in the SRs for few benchmark points in our model corresponding to $\left(m_{H^{ \pm}}, m_{N}\right)=$ $(200,10)$, and $(200,142) \mathrm{GeV}$.

- The expected number of background events $\left(N_{b}\right)$ is generated randomly from a Gaussian distribution where its mean is $N_{\mathrm{b}}$ and its standard deviation is $\Delta n_{\mathrm{b}}$. The actual background events number $\left(\tilde{N}_{\mathrm{b}}\right)$ is obtained by using $N_{b}$ as parameter from Poisson distribution. Hence, the background probability $p_{\mathrm{b}}$ reads:

$$
p_{\mathrm{b}}=\frac{N_{\text {toys }}\left(\tilde{N}_{\mathrm{b}} \leq n_{\mathrm{obs}}\right)}{N_{\text {toys }}}
$$

where $N_{\text {toys }}\left(\tilde{N}_{\mathrm{b}} \leq n_{\text {obs }}\right),{ }^{7}$ illustrates the toy experiments number in which the condition $\tilde{N}_{\mathrm{b}} \leq n_{\text {obs }}$ is verified.

- The second step consists of computing the signal-plus-background probability $p_{\mathrm{b}+\mathrm{s}}$. To get the latter probability, the actual number of signal-plus-background events $\left(\tilde{N}_{\mathrm{s}}+\tilde{N}_{\mathrm{b}}\right)$ is generated randomly following a Poisson distribution using the $n_{s}+N_{b}$ parameter. Therefore, in this case the probability, $p_{\mathrm{b}+\mathrm{s}}$, can be defined by

$$
p_{\mathrm{b}+\mathrm{s}}=\frac{N_{\mathrm{toys}}\left(\tilde{N}_{\mathrm{s}}+\tilde{N}_{\mathrm{b}} \leq n_{\mathrm{obs}}\right)}{N_{\text {toys }}}
$$

with $N_{\text {toys }}\left(\tilde{N}_{\mathrm{s}}+\tilde{N}_{\mathrm{b}} \leq n_{\text {obs }}\right)$ represents the number of toy experiments ${ }^{8}$ in which the condition $\tilde{N}_{\mathrm{s}}+\tilde{N}_{\mathrm{b}} \leq n_{\mathrm{obs}}$ is satisfied.

The obtained values of the background probability $\left(p_{\mathrm{b}}\right)$ and the signal-plus-background probability $\left(p_{\mathrm{b}+\mathrm{s}}\right)$ in (B.2) and (B.3), respectively, are then used to compute the $\mathrm{CL}_{s}$ which

\footnotetext{
${ }^{7}$ We note that only the number of toy experiments yielding positive $N_{b}$ are retained.

${ }^{8}$ We note that only the number of toy experiments yielding positive $N_{b}+n_{s}$ are retained.
} 


\begin{tabular}{|ccccc|}
\hline Region & $\begin{array}{c}\text { SR-SF-0J } \\
\in[100, \infty)\end{array}$ & $\begin{array}{c}\text { SR-SF-0J } \\
\in[160, \infty)\end{array}$ & $\begin{array}{c}\text { SR-SF-0J } \\
\in[100,120)\end{array}$ & $\begin{array}{c}\text { SR-SF-0J } \\
\in[120,160)\end{array}$ \\
\hline Backgrounds (Linear) & $3107 \pm 258$ & $805 \pm 64$ & $1208 \pm 129$ & $1100 \pm 107$ \\
\hline Backgrounds (Poisson) & $3107 \pm 55$ & $805 \pm 13$ & $1208 \pm 27$ & $1100 \pm 23$ \\
\hline$m_{H^{ \pm}}, m_{N}=(500,10) \mathrm{GeV}$ & 125.2 & 105.7 & 6.5 & 12.9 \\
$m_{H^{ \pm}}, m_{N}=(500,425) \mathrm{GeV}$ & 12.9 & 0.06 & 8.6 & 4.3 \\
\hline Region & SR-SF-1J & SR-SF-1J & SR-SF-1J & SR-SF-1J \\
$M_{T 2}(\mathrm{GeV})$ & $\in[100, \infty)$ & $\in[160, \infty)$ & $\in[100,120)$ & $\in[120,160)$ \\
\hline Backgrounds (Linear) & $2676 \pm 259$ & $777 \pm 108$ & $1036 \pm 172$ & $863 \pm 86$ \\
\hline Backgrounds (Poisson) & $2676 \pm 55$ & $777 \pm 23$ & $1036 \pm 37$ & $863 \pm 18$ \\
\hline$m_{H^{ \pm}}, m_{N}=(500,10) \mathrm{GeV}$ & 88.5 & 75.5 & 4.3 & 8.6 \\
$m_{H^{ \pm}}, m_{N}=(500,425) \mathrm{GeV}$ & 8.6 & 0.2 & 4.3 & 4.3 \\
\hline
\end{tabular}

Table 4. Expected background event yields, at $\mathcal{L}=3000 \mathrm{fb}^{-1}$, for the 'same flavor' (SF) inclusive signal regions SRs estimated by extrapolating the results in table 3. The uncertainties on the background yields are computed by extrapolating the errors at the $\mathrm{LHC}_{139 \mathrm{fb}^{-1}}$ linearly (assuming they are dominated by systematic uncertainties) or according to a Poisson distribution (assuming they are dominated by statistics). For reference, we show the number of events in the SRs for few benchmark points in our model corresponding to $\left(m_{H^{ \pm}}, m_{N}\right)=(500,10)$, and $(500,425) \mathrm{GeV}$.

reads as

$$
\mathrm{CL}_{s}=\max \left(0,1-\frac{p_{\mathrm{b}+\mathrm{s}}}{p_{\mathrm{b}}}\right) .
$$

In figure 9, we display the exclusion contours projected on the charged Higgs mass $\left(m_{H^{ \pm}}\right)$, and the Majorana DM mass, $\left(m_{N}\right)$, for all inclusive signal regions. It can clearly be seen that the SR-SF-0J implies more constraining power compared to SR-SF-1J category. Therefore, the strongest exclusion comes from the SR-SF-0J with $M_{T 2} \in[160, \infty)$ which rules out the charged Higgs masses up to $380 \mathrm{GeV}$. As matter of fact, the search itself does not constrain small mass splittings; i.e. $m_{H^{ \pm}}-m_{N}<50 \mathrm{GeV}$.

In our work we use MadAnalysis [95] to cope with these limits at the expected luminosity $\mathcal{L}=3000 \mathrm{fb}^{-1}$. First, the number of expected background events in this case is calculated as:

$$
n_{\mathrm{b}}^{3000}=\frac{3000}{139} n_{\mathrm{b}}=21.58 n_{\mathrm{b}} .
$$

Moreoever, the number of observed events $\left(n_{\text {obs }}\right)$ will be set to the extrapolated background event yields, i.e. $n_{\mathrm{obs}}^{3000}=n_{\mathrm{b}}^{3000}$. Consequently, the extrapolated errors can be computed as:

$$
\Delta n_{\mathrm{b}}^{3000}=21.58 \Delta_{\mathrm{b}, \mathrm{syst}} \oplus 4.64 \Delta_{\mathrm{b}, \mathrm{stat}} .
$$

Table 4 lists the extrapolated event yields for the backgrounds and the signals. One should mention that the uncertainties on the background contributions are assumed to be dominated by just the statistical errors. 



Figure 9. The $\mathrm{CL}_{s}$ at $95 \% \mathrm{CL}$ projected on the mass of the charged Higgs mass $\left(m_{H^{ \pm}}\right)$and the mass of the Majorana DM $\left(m_{N}\right)$ for different signal regions for the SR-SF-0J and SR-SF-1J categories. The exclusions are shown for different Inclusive SRs which depend on the range of the $M_{T 2}$ variable. The shaded light navy area is forbidden by the constraint $m_{H^{ \pm}}>m_{N}$ required by DM stability. 
Open Access. This article is distributed under the terms of the Creative Commons Attribution License (CC-BY 4.0), which permits any use, distribution and reproduction in any medium, provided the original author(s) and source are credited.

\section{References}

[1] G. Bertone, D. Hooper and J. Silk, Particle dark matter: Evidence, candidates and constraints, Phys. Rept. 405 (2005) 279 [hep-ph/0404175] [INSPIRE].

[2] PlancK collaboration, Planck 2015 results. XIII. Cosmological parameters, Astron. Astrophys. 594 (2016) A13 [arXiv:1502.01589] [INSPIRE].

[3] XENON collaboration, Dark Matter Search Results from a One Ton-Year Exposure of XENON1T, Phys. Rev. Lett. 121 (2018) 111302 [arXiv:1805.12562] [InSPIRE].

[4] PandaX-II collaboration, Dark Matter Results From 54-Ton-Day Exposure of PandaX-II Experiment, Phys. Rev. Lett. 119 (2017) 181302 [arXiv:1708.06917] [InSPIRE].

[5] PAMELA collaboration, PAMELA results on the cosmic-ray antiproton flux from $60 \mathrm{MeV}$ to $180 \mathrm{GeV}$ in kinetic energy, Phys. Rev. Lett. 105 (2010) 121101 [arXiv:1007.0821] [InSPIRE].

[6] AMS collaboration, First Result from the Alpha Magnetic Spectrometer on the International Space Station: Precision Measurement of the Positron Fraction in Primary Cosmic Rays of 0.5-350 GeV, Phys. Rev. Lett. 110 (2013) 141102 [InSPIRE].

[7] MAGIC and Fermi-LAT collaborations, Limits to Dark Matter Annihilation Cross-Section from a Combined Analysis of MAGIC and Fermi-LAT Observations of Dwarf Satellite Galaxies, JCAP 02 (2016) 039 [arXiv:1601.06590] [INSPIRE].

[8] H.E.S.S. collaboration, Search for dark matter annihilations towards the inner Galactic halo from 10 years of observations with H.E.S.S, Phys. Rev. Lett. 117 (2016) 111301 [arXiv: 1607.08142] [INSPIRE].

[9] ATLAS collaboration, Overview of dark matter searches at the ATLAS experiment, Int. J. Mod. Phys. Conf. Ser. 43 (2016) 1660196 [InSPIRE].

[10] CMS collaboration, Searches for Dark Matter with CMS, PoS LHCP2018 (2018) 284 [INSPIRE].

[11] XENON collaboration, Projected WIMP sensitivity of the XENONnT dark matter experiment, JCAP 11 (2020) 031 [arXiv:2007.08796] [INSPIRE].

[12] R.K. Leane, T.R. Slatyer, J.F. Beacom and K.C.Y. Ng, GeV-scale thermal WIMPs: Not even slightly ruled out, Phys. Rev. D 98 (2018) 023016 [arXiv: 1805.10305] [INSPIRE].

[13] V. Silveira and A. Zee, SCALAR PHANTOMS, Phys. Lett. B 161 (1985) 136 [INSPIRE].

[14] C.P. Burgess, M. Pospelov and T. ter Veldhuis, The Minimal model of nonbaryonic dark matter: A Singlet scalar, Nucl. Phys. B 619 (2001) 709 [hep-ph/0011335] [INSPIRE].

[15] G. Arcadi, C. Gross, O. Lebedev, S. Pokorski and T. Toma, Evading Direct Dark Matter Detection in Higgs Portal Models, Phys. Lett. B 769 (2017) 129 [arXiv:1611.09675] [INSPIRE].

[16] J.A. Casas, D.G. Cerdeño, J.M. Moreno and J. Quilis, Reopening the Higgs portal for single scalar dark matter, JHEP 05 (2017) 036 [arXiv:1701.08134] [INSPIRE]. 
[17] E. Ma, Pathways to naturally small neutrino masses, Phys. Rev. Lett. 81 (1998) 1171 [hep-ph/9805219] [INSPIRE].

[18] L.M. Krauss, S. Nasri and M. Trodden, A Model for neutrino masses and dark matter, Phys. Rev. D 67 (2003) 085002 [hep-ph/0210389] [INSPIRE].

[19] M. Aoki, S. Kanemura and O. Seto, Neutrino mass, Dark Matter and Baryon Asymmetry via TeV-Scale Physics without Fine-Tuning, Phys. Rev. Lett. 102 (2009) 051805 [arXiv: 0807.0361] [INSPIRE].

[20] M. Gustafsson, J.M. No and M.A. Rivera, Predictive Model for Radiatively Induced Neutrino Masses and Mixings with Dark Matter, Phys. Rev. Lett. 110 (2013) 211802 [Erratum ibid. 112 (2014) 259902] [arXiv:1212.4806] [INSPIRE].

[21] S.M. Boucenna, S. Morisi and J.W.F. Valle, The low-scale approach to neutrino masses, Adv. High Energy Phys. 2014 (2014) 831598 [arXiv:1404.3751] [INSPIRE].

[22] Y. Cai, J. Herrero-García, M.A. Schmidt, A. Vicente and R.R. Volkas, From the trees to the forest: a review of radiative neutrino mass models, Front. in Phys. 5 (2017) 63 [arXiv: 1706.08524] [INSPIRE].

[23] B. Swiezewska and M. Krawczyk, Diphoton rate in the inert doublet model with a $125 \mathrm{GeV}$ Higgs boson, Phys. Rev. D 88 (2013) 035019 [arXiv:1212.4100] [INSPIRE].

[24] A. Arhrib, R. Benbrik and N. Gaur, $H \rightarrow \gamma \gamma$ in Inert Higgs Doublet Model, Phys. Rev. D 85 (2012) 095021 [arXiv:1201.2644] [INSPIRE].

[25] A. Jueid, J. Kim, S. Lee, S.Y. Shim and J. Song, Phenomenology of the Inert Doublet Model with a global U(1) symmetry, Phys. Rev. D 102 (2020) 075011 [arXiv:2006.10263] [INSPIRE].

[26] ATLAS and CMS collaborations, Measurements of the Higgs boson production and decay rates and constraints on its couplings from a combined ATLAS and CMS analysis of the LHC pp collision data at $\sqrt{s}=7$ and $8 \mathrm{TeV}, \mathrm{JHEP} 08$ (2016) 045 [arXiv:1606.02266] [INSPIRE].

[27] CMS collaboration, Projected performance of Higgs analyses at the HL-LHC for ECFA 2016, Tech. Rep. CMS-PAS-FTR-16-002 (2017).

[28] O. Cerri, M. de Gruttola, M. Pierini, A. Podo and G. Rolandi, Study the effect of beam energy spread and detector resolution on the search for Higgs boson decays to invisible particles at a future $e^{+} e^{-}$circular collider, Eur. Phys. J. C 77 (2017) 116 [arXiv:1605.00100] [InSPIRE].

[29] D.M. Asner et al., ILC Higgs White Paper, in Community Summer Study 2013: Snowmass on the Mississippi, Minneapolis, MN, U.S.A., 29 July-6 August 2013 [arXiv:1310.0763] [INSPIRE].

[30] CEPC Study Group collaboration, CEPC Conceptual Design Report: Volume 2 Physics $\mathcal{E}$ Detector, arXiv:1811.10545 [INSPIRE].

[31] M. Selvaggi, Higgs measurements at the FCC-hh, PoS ICHEP2018 (2019) 684 [INSPIRE].

[32] A. Arhrib, R. Benbrik, J. El Falaki and A. Jueid, Radiative corrections to the Triple Higgs Coupling in the Inert Higgs Doublet Model, JHEP 12 (2015) 007 [arXiv:1507.03630] [INSPIRE].

[33] G.C. Branco, P.M. Ferreira, L. Lavoura, M.N. Rebelo, M. Sher and J.P. Silva, Theory and phenomenology of two-Higgs-doublet models, Phys. Rept. 516 (2012) 1 [arXiv:1106.0034] [INSPIRE]. 
[34] S. Kanemura, T. Kubota and E. Takasugi, Lee-Quigg-Thacker bounds for Higgs boson masses in a two doublet model, Phys. Lett. B 313 (1993) 155 [hep-ph/9303263] [INSPIRE].

[35] A.G. Akeroyd, A. Arhrib and E.-M. Naimi, Note on tree level unitarity in the general two Higgs doublet model, Phys. Lett. B 490 (2000) 119 [hep-ph/0006035] [INSPIRE].

[36] I.F. Ginzburg, K.A. Kanishev, M. Krawczyk and D. Sokolowska, Evolution of Universe to the present inert phase, Phys. Rev. D 82 (2010) 123533 [arXiv:1009.4593] [InSPIRE].

[37] T. Hahn, Automatic loop calculations with FeynArts, FormCalc, and LoopTools, Nucl. Phys. B Proc. Suppl. 89 (2000) 231 [hep-ph/0005029] [INSPIRE].

[38] T. Hahn, Generating Feynman diagrams and amplitudes with FeynArts 3, Comput. Phys. Commun. 140 (2001) 418 [hep-ph/0012260] [INSPIRE].

[39] CMS collaboration, Search for invisible decays of a Higgs boson produced through vector boson fusion in proton-proton collisions at $\sqrt{s}=13$ TeV, Phys. Lett. B 793 (2019) 520 [arXiv: 1809.05937] [INSPIRE].

[40] G. Passarino and M.J.G. Veltman, One Loop Corrections for $e^{+} e^{-}$Annihilation Into $\mu^{+} \mu^{-}$ in the Weinberg Model, Nucl. Phys. B 160 (1979) 151 [InSPIRE].

[41] OPAL collaboration, Search for anomalous production of dilepton events with missing transverse momentum in $e^{+} e^{-}$collisions at $\sqrt{s}=183$ Gev to 209-GeV, Eur. Phys. J. C 32 (2004) 453 [hep-ex/0309014] [INSPIRE].

[42] A. Ahriche, A. Arhrib, A. Jueid, S. Nasri and A. de La Puente, Mono-Higgs Signature in the Scotogenic Model with Majorana Dark Matter, Phys. Rev. D 101 (2020) 035038 [arXiv: 1811.00490] [INSPIRE].

[43] N. Blinov, J. Kozaczuk, D.E. Morrissey and A. de la Puente, Compressing the Inert Doublet Model, Phys. Rev. D 93 (2016) 035020 [arXiv:1510.08069] [InSPIRE].

[44] MEG collaboration, New constraint on the existence of the $\mu^{+} \rightarrow e^{+} \gamma$ decay, Phys. Rev. Lett. 110 (2013) 201801 [arXiv: 1303.0754] [INSPIRE].

[45] BABAR collaboration, Searches for Lepton Flavor Violation in the Decays $\tau^{ \pm} \rightarrow e^{ \pm} \gamma$ and $\tau^{ \pm} \rightarrow \mu^{ \pm} \gamma$, Phys. Rev. Lett. 104 (2010) 021802 [arXiv:0908.2381] [INSPIRE].

[46] T. Toma and A. Vicente, Lepton Flavor Violation in the Scotogenic Model, JHEP 01 (2014) 160 [arXiv:1312.2840] [INSPIRE].

[47] A. Ahriche, A. Jueid and S. Nasri, Radiative neutrino mass and Majorana dark matter within an inert Higgs doublet model, Phys. Rev. D 97 (2018) 095012 [arXiv:1710.03824] [INSPIRE].

[48] ATLAS collaboration, Search for electroweak production of charginos and sleptons decaying into final states with two leptons and missing transverse momentum in $\sqrt{s}=13 \mathrm{TeV} p p$ collisions using the ATLAS detector, Eur. Phys. J. C 80 (2020) 123 [arXiv:1908.08215] [INSPIRE].

[49] J. Alwall et al., The automated computation of tree-level and next-to-leading order differential cross sections, and their matching to parton shower simulations, JHEP 07 (2014) 079 [arXiv: 1405.0301] [INSPIRE].

[50] C. Degrande, C. Duhr, B. Fuks, D. Grellscheid, O. Mattelaer and T. Reiter, UFO - The Universal FeynRules Output, Comput. Phys. Commun. 183 (2012) 1201 [arXiv:1108.2040] [INSPIRE]. 
[51] NNPDF collaboration, Parton distributions for the LHC Run II, JHEP 04 (2015) 040 [arXiv: 1410.8849] [INSPIRE].

[52] A. Buckley et al., LHAPDF6: parton density access in the LHC precision era, Eur. Phys. J. $C 75$ (2015) 132 [arXiv:1412.7420] [INSPIRE].

[53] P. Artoisenet, R. Frederix, O. Mattelaer and R. Rietkerk, Automatic spin-entangled decays of heavy resonances in Monte Carlo simulations, JHEP 03 (2013) 015 [arXiv:1212.3460] [INSPIRE].

[54] M.L. Mangano, M. Moretti, F. Piccinini and M. Treccani, Matching matrix elements and shower evolution for top-quark production in hadronic collisions, JHEP 01 (2007) 013 [hep-ph/0611129] [INSPIRE].

[55] T. Sjöstrand et al., An introduction to PYTHIA 8.2, Comput. Phys. Commun. 191 (2015) 159 [arXiv: 1410.3012] [INSPIRE].

[56] M. Cacciari, G.P. Salam and G. Soyez, FastJet User Manual, Eur. Phys. J. C 72 (2012) 1896 [arXiv: 1111.6097] [INSPIRE].

[57] M. Cacciari, G.P. Salam and G. Soyez, The anti- $k_{t}$ jet clustering algorithm, JHEP 04 (2008) 063 [arXiv: 0802.1189] [INSPIRE].

[58] C.G. Lester and D.J. Summers, Measuring masses of semiinvisibly decaying particles pair produced at hadron colliders, Phys. Lett. B 463 (1999) 99 [hep-ph/9906349] [INSPIRE].

[59] A. Barr, C. Lester and P. Stephens, m(T2): The Truth behind the glamour, J. Phys. G 29 (2003) 2343 [hep-ph/0304226] [INSPIRE].

[60] E. Conte, B. Fuks and G. Serret, MadAnalysis 5, A User-Friendly Framework for Collider Phenomenology, Comput. Phys. Commun. 184 (2013) 222 [arXiv:1206.1599] [InSPIRE].

[61] E. Conte, B. Dumont, B. Fuks and C. Wymant, Designing and recasting LHC analyses with MadAnalysis 5, Eur. Phys. J. C 74 (2014) 3103 [arXiv: 1405.3982] [InSPIRE].

[62] E. Conte and B. Fuks, Confronting new physics theories to LHC data with MADANALYSIS 5, Int. J. Mod. Phys. A 33 (2018) 1830027 [arXiv:1808.00480] [INSPIRE].

[63] J.Y. Araz et al., Proceedings of the second MadAnalysis 5 workshop on LHC recasting in Korea, Mod. Phys. Lett. A 36 (2021) 2102001 [arXiv:2101.02245] [inSPIRE].

[64] J.Y. Araz and B. Fuks, Implementation of the ATLAS-SUSY-2018-32 analysis (sleptons and electroweakinos with two leptons and missing transverse energy; $\left.139 \mathrm{fb}^{-1}\right)$, Mod. Phys. Lett. A 36 (2021) 2141005 [INSPIRE].

[65] A.L. Read, Presentation of search results: The $C L_{s}$ technique, J. Phys. G 28 (2002) 2693 [INSPIRE].

[66] K. Kong and K.T. Matchev, Precise calculation of the relic density of Kaluza-Klein dark matter in universal extra dimensions, JHEP 01 (2006) 038 [hep-ph/0509119] [INSPIRE].

[67] M. Cirelli, E. Del Nobile and P. Panci, Tools for model-independent bounds in direct dark matter searches, JCAP 10 (2013) 019 [arXiv:1307.5955] [INSPIRE].

[68] R.J. Hill and M.P. Solon, Standard Model anatomy of WIMP dark matter direct detection II: QCD analysis and hadronic matrix elements, Phys. Rev. D 91 (2015) 043505 [arXiv: 1409.8290] [INSPIRE].

[69] F. Bishara, J. Brod, B. Grinstein and J. Zupan, From quarks to nucleons in dark matter direct detection, JHEP 11 (2017) 059 [arXiv:1707.06998] [INSPIRE]. 
[70] J. Ellis, N. Nagata and K.A. Olive, Uncertainties in WIMP Dark Matter Scattering Revisited, Eur. Phys. J. C 78 (2018) 569 [arXiv:1805.09795] [InSPIRE].

[71] M. Backovic, K. Kong and M. McCaskey, MadDM v.1.0: Computation of Dark Matter Relic Abundance Using MadGraph5, Phys. Dark Univ. 5-6 (2014) 18 [arXiv:1308.4955] [INSPIRE].

[72] M. Backović, A. Martini, O. Mattelaer, K. Kong and G. Mohlabeng, Direct Detection of Dark Matter with MadDM v.2.0, Phys. Dark Univ. 9-10 (2015) 37 [arXiv:1505.04190] [INSPIRE].

[73] F. Ambrogi et al., MadDM v.3.0: a Comprehensive Tool for Dark Matter Studies, Phys. Dark Univ. 24 (2019) 100249 [arXiv: 1804.00044] [INSPIRE].

[74] FERMI-LAT collaboration, Updated search for spectral lines from Galactic dark matter interactions with pass 8 data from the Fermi Large Area Telescope, Phys. Rev. D 91 (2015) 122002 [arXiv: 1506.00013] [INSPIRE].

[75] H. Baer et al., eds., The International Linear Collider Technical Design Report - Volume 2: Physics, arXiv:1306.6352 [INSPIRE].

[76] J.L. Feng, Physics at e-e-colliders, Int. J. Mod. Phys. A 15 (2000) 2355 [hep-ph/0002055] [INSPIRE].

[77] C.A. Heusch, The International Linear Collider in its electron-electron version, Int. J. Mod. Phys. A 20 (2005) 7289 [inSPIRE].

[78] H. Abouabid et al., One-loop radiative corrections to $e^{+} e^{-} \rightarrow Z h^{0} / H^{0} A^{0}$ in the Inert Higgs Doublet Model, arXiv:2009.03250 [INSPIRE].

[79] C. Bartels, M. Berggren and J. List, Characterising WIMPs at a future $e^{+} e^{-}$Linear Collider, Eur. Phys. J. C 72 (2012) 2213 [arXiv:1206.6639] [INSPIRE].

[80] J.R. Andersen, M. Rauch and M. Spannowsky, Dark Sector spectroscopy at the ILC, Eur. Phys. J. C 74 (2014) 2908 [arXiv: 1308.4588] [InSPIRE].

[81] A. Ahriche, S. Nasri and R. Soualah, Radiative neutrino mass model at the $e^{-} e^{+}$linear collider, Phys. Rev. D 89 (2014) 095010 [arXiv: 1403.5694] [InSPIRE].

[82] P. Ko and H. Yokoya, Search for Higgs portal DM at the ILC, JHEP 08 (2016) 109 [arXiv: 1603.04737] [INSPIRE].

[83] N. Baouche and A. Ahriche, Identifying the nature of dark matter at $e^{-} e^{+}$colliders, Phys. Rev. D 96 (2017) 055029 [arXiv: 1707.05263] [INSPIRE].

[84] J. Kalinowski, W. Kotlarski, T. Robens, D. Sokolowska and A.F. Zarnecki, Exploring Inert Scalars at CLIC, JHEP 07 (2019) 053 [arXiv: 1811.06952] [INSPIRE].

[85] D.K. Ghosh, T. Katayose, S. Matsumoto, I. Saha, S. Shirai and T. Tanabe, Role of future lepton colliders for fermionic Z-portal dark matter models, Phys. Rev. D 101 (2020) 015007 [arXiv: 1906.06864] [INSPIRE].

[86] S. Baum, P. Sandick and P. Stengel, Hunting for scalar lepton partners at future electron colliders, Phys. Rev. D 102 (2020) 015026 [arXiv: 2004.02834] [InSPIRE].

[87] DELPHES 3 collaboration, DELPHES 3, A modular framework for fast simulation of a generic collider experiment, JHEP 02 (2014) 057 [arXiv: 1307.6346] [INSPIRE].

[88] M. Aoki and S. Kanemura, Probing the Majorana nature of TeV-scale radiative seesaw models at collider experiments, Phys. Lett. B 689 (2010) 28 [arXiv:1001.0092] [InSPIRE].

[89] A. Jueid and S. Nasri, Dark Matter meets Unification, to be published (2021). 
[90] J.D. Hunter, Matplotlib: A 2D Graphics Environment, Comput. Sci. Eng. 9 (2007) 90 [INSPIRE].

[91] C.R. Harris et al., Array programming with NumPy, Nature 585 (2020) 357 [arXiv:2006.10256] [INSPIRE].

[92] P. Virtanen et al., SciPy 1.0: Fundamental Algorithms for Scientific Computing in Python, Nature Meth. 17 (2020) 261 [arXiv:1907.10121] [INSPIRE].

[93] H.H. Patel, Package-X: A Mathematica package for the analytic calculation of one-loop integrals, Comput. Phys. Commun. 197 (2015) 276 [arXiv:1503.01469] [INSPIRE].

[94] N. Okada and T. Yamada, Simple fermionic dark matter models and Higgs boson couplings, JHEP 10 (2013) 017 [arXiv: 1304.2962] [InSPIRE].

[95] J.Y. Araz, M. Frank and B. Fuks, Reinterpreting the results of the LHC with MadAnalysis 5: uncertainties and higher-luminosity estimates, Eur. Phys. J. C 80 (2020) 531 [arXiv: 1910.11418] [INSPIRE]. 